\title{
3D Human Microvessel-on-a-Chip Model for Studying Monocyte-to-Endothelium Adhesion under Flow - Application in Systems Toxicology
}

\author{
Carine Poussin 1\#, Bart Kramer2\#, Henriette L. Lanz 2\#, Angelique van den Heuvel ${ }^{2}$, Alexandra Laurent', \\ Thomas Olivier2, Marjolein Vermeer², Dariusz Peric', Karine Baumer', Rémi Dulize', Emmanuel Guedj', \\ Nikolai V. Ivanov' ${ }^{1}$, Manuel C. Peitsch', Julia Hoeng' ${ }^{1}$ and Jos Joore 2 \\ ${ }^{1}$ PMI R\&D, Philip Morris Products S.A., Neuchâtel, Switzerland; ${ }^{2}$ MIMETAS BV, Leiden, The Netherlands
}

\begin{abstract}
Lifestyle and genetic factors can lead to the development of atherosclerosis and, ultimately, cardiovascular adverse events. Rodent models are commonly used to investigate mechanism(s) of atherogenesis. However, the 3R principles, aiming to limit animal testing, encourage the scientific community to develop new, physiologically relevant alternatives. Leveraging the 96-chip OrganoPlate ${ }^{\circledR}$, a microfluidic plafform, we have established a three-dimensional (3D) model of endothelial microvessels-on-a-chip under flow using primary human coronary arterial endothelial cells. As functional readout, we have set up an assay to measure the adhesion of monocytes to the lumen of perfused microvessels. For monitoring molecular changes in microvessels, we have established the staining and quantification of specific protein markers of inflammation and oxidative stress using high-content imaging as well as analyzed transcriptome changes using microarrays. To demonstrate its usefulness in systems toxicology, we used our 3D vasculature-on-a-chip model to assess the impact of the Tobacco Heating System (THS) 2.2, a candidate modified-risk tobacco product, and the 3R4F reference cigarette on the adhesion of monocytic cells to endothelial microvessels. Our results show that THS 2.2 aerosolconditioned medium had a reduced effect on monocyte-endothelial adhesion compared with that of 3R4F smoke-conditioned medium. In conclusion, we have established a relevant 3D vasculature-on-a-chip model for investigating leukocyte-endothelial microvessel adhesion. A case study illustrates how the model can be used for product testing in the context of systems toxicology-based risk assessment. The current model and its potential further development options also open perspectives of applications in vascular disease research and drug discovery.
\end{abstract}

\section{Introduction}

The endothelium is a single layer of cells at the interface between circulating blood and organ tissues, which plays critical roles in vascular processes, such as barrier permeability (Radeva and Waschke, 2018), vasotone regulation, leukocyte adhesiveness and extravasation, blood clotting and angiogenesis (Favero et al., 2014). Endothelial dysfunction, which increases permeability, adhesiveness and transmigration of leukocytes as well as the accumulation of fatty streaks in the subendothelial compartment (Insull, 2009), is a hallmark of atherosclerosis, characterized by the development of plaques that can become unstable and rupture, resulting in adverse cardiovascular events (Favero et al., 2014).
The development of atherosclerosis and its prevention by therapeutic interventions is studied extensively in humans and in rodent models. Although highly relevant, the use of in vivo models remains technically challenging for in-depth and rapid mechanistic investigations, such as the deconvolution of the effects of different molecules present in a milieu on endothelial cells. Moreover, the identification of potent drug compounds for therapeutic intervention and the testing of compounds/products for toxicological safety or risk assessments require high-throughput settings for a pre-screening phase that is generally not conducted in vivo. The perspective of the $3 \mathrm{R}$ principles, "replacement, reduction, and refinement," which aim to limit animal testing (Burden et al., 2015), encourages the scientific community to develop

\# contributed equally

Received November 30, 2018; Accepted August 15, 2019;

Epub August 22, 2019; (C) The Authors, 2019.

This is an Open Access article distributed under the terms of the Creative Commons Attribution 4.0 International license (http://creativecommons.org/licenses/by/4.0/), which permits unrestricted use, distribution and reproduction in any medium, proviALTEX 37(1), 047-063. doi:10.14573/altex.1811301 ded the original work is appropriately cited.

Correspondence: Carine Poussin, $\mathrm{PhD}$

PMI R\&D, Philip Morris Products S.A.

Quai Jeanrenaud 5, 2000 Neuchâtel, Switzerland

(Carine.Poussin@pmi.com) 


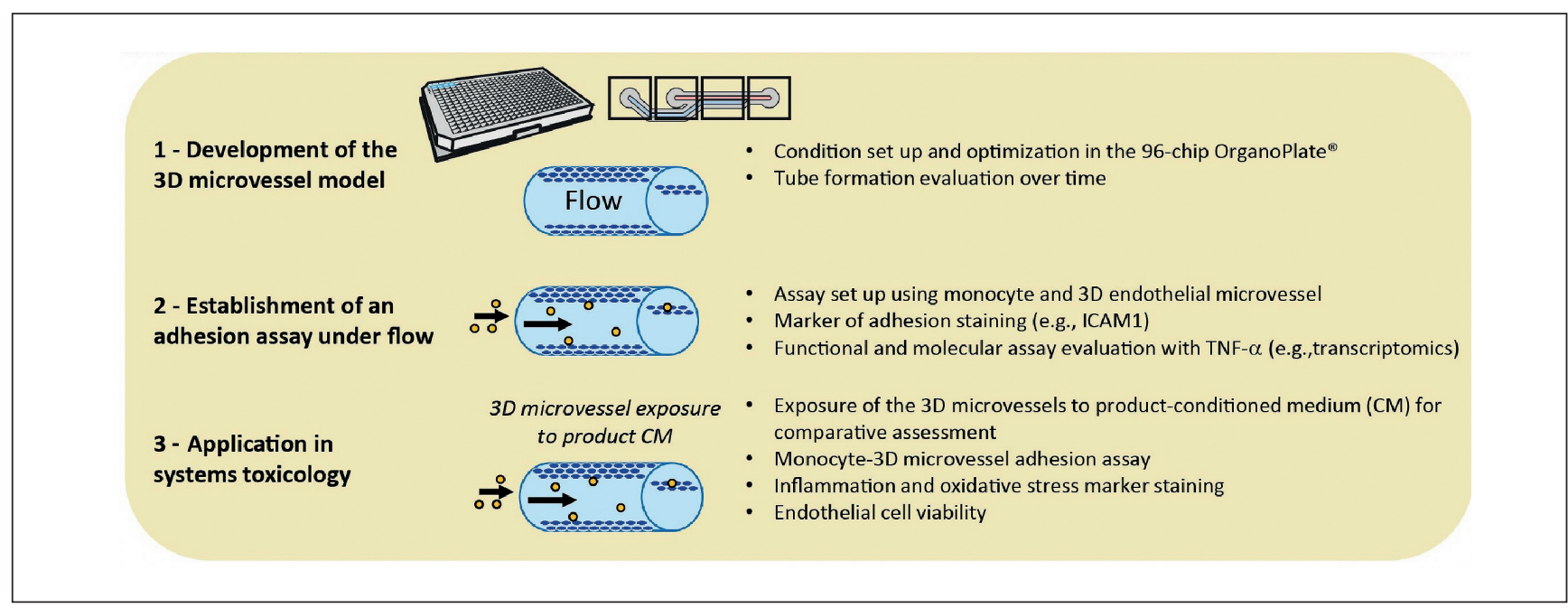

Fig. 1: Overview of the steps for the development, functional evaluation, and real-case application in systems toxicology of our 3D human microvessel-on-a-chip model for studying monocyte-endothelial adhesion under flow

relevant in vitro alternatives. So far, two-dimensional (2D) and static endothelial in vitro models have been used extensively, but more physiologically relevant models are required that integrate the three-dimensional (3D) geometry of vessels and hemodynamic flow. These properties play important mechanical roles through mechano-transduction signaling, which influences vascular differentiation and proliferation as well as endothelial cell morphology and responses to stimuli (Lee et al., 2015; Chatterjee, 2018). Along with other factors, flow patterns and the magnitude of shear stress promote anti- or pro-atherogenic effects on endothelial cells that contribute to the protection from or promotion of vascular diseases (Hsieh et al., 2014; Chatterjee, 2018).

The emergence of a wide range of engineered microfluidic systems and techniques enabling the culture of cells under flow and in a 3D space has accelerated the innovation of advanced, perfusable in vitro vascular models. These models vary in complexity and physiological relevance from a simple planar endothelial cell layer on top of which controlled shear stress can be applied to 3D microvessels or even more complex vasculature networks (Bogorad et al., 2015; van Duinen et al., 2017). Models are classified according to their method of fabrication and constitute a toolbox for studying different aspects of vascular biology (Bogorad et al., 2015). Each model offers advantages (e.g., high physiological relevance, high-throughput capacity, easy setup) and possesses limitations (e.g., time-consuming to manufacture, low throughput, lower physiological relevance) (Bogorad et al., 2015). Therefore, it is important to define the biological mechanism(s) of interest, the extension potential of the model, and the capacity needed to select or develop a vascular model that adequately balances ex- perimental feasibility, physiological relevance, and throughput capacity for larger experimental condition screening.

Lifestyle and genetic predisposition affect the development of vascular disorders that result in atherogenesis (Nahrendorf and Swirski, 2015). Smoking, for instance, is a recognized major risk factor for the development of cardiovascular diseases (Messner and Bernhard, 2014). Over time, smokers develop low-grade inflammation and oxidative properties in the systemic compartment that can alter endothelial function, resulting in the initiation and progression of atherosclerosis (Yanbaeva et al., 2007). The health risks associated with cigarette smoking are attributable to toxicants that are generated in the smoke through tobacco combustion (Surgeon General, 2010). Therefore, reducing exposure to smoke constituents is crucial for risk reduction (Murphy et al., 2017), for which the development of modified risk tobacco products (MRTP) is necessary (Food and Drug Administration, 2012). Potential MRTPs that heat tobacco rather than burning it have been developed, offering significant decreases in the complexity and number of toxicants generated in the aerosol during use (Schaller et al., 2016). Pre-clinical and clinical studies aimed to assess these products have compared them with cigarettes to determine whether this reduction in exposure translates into a reduction in health risk in both laboratory models and humans (Murphy et al., 2017). Overall, the results indicated that the Tobacco Heating System (THS) 2.2, a candidate MRTP, and the Carbon-Heated Tobacco Product (CHTP) 1.2, a potential MRTP, exert reduced effects on respiratory and cardiovascular functional and molecular endpoints in both in vitro and in vivo laboratory models (Phillips et al., 2016; Poussin et al., 2016, 2018; van der Toorn et al.,

2D, two-dimensional; 3D, three-dimensional; ANOVA, analysis of variance; ECM, extracellular matrix; FBS, fetal bovine serum; FC, fold-change; FDR, false discovery rate FITC, fluorescein isothiocyanate; fRMA, frozen robust microarray analysis; GSH, glutathione; HBSS, Hank's Balanced Salt Solution; HCAEC, human coronary artery endothelial cell; ICAM1, intercellular adhesion molecule 1; MM6, Mono-Mac-6; MRTP, modified risk tobacco product; NPA, network perturbation amplitude; PC, principal component; PCA, principal component analysis; RPMI, Roswell Park Memorial Institute; SRP, systems response profile; THS, Tobacco Heating System; TNFa, tumor necrosis factor alpha; TRITC, tetramethylrhodamine isothiocyanate; VC, vehicle control; WST-8, water-soluble tetrazolium salt 
2015). Human studies showed a significant reduction in biomarkers of exposure and potential harm after switching from cigarettes to THS 2.2 (Ludicke et al., 2018a,b).

The present work aimed to develop a 3D vasculature-on-a-chip model under flow to study monocyte-endothelium adhesion and provide a use case application by applying this model to tobacco product testing in a systems toxicology framework (Fig. 1). Using the OrganoPlate ${ }^{\circledR}$, a standardized 96-chip microfluidic cell culture plate, we optimized conditions to grow endothelial microvessels using disease-relevant primary human coronary artery endothelial cells (HCAEC, Fig. 1, Step 1). This microfluidic platform has been used previously to develop perfusable human umbilical vein endothelial cells, VeraVec human endothelial cell vessels, to study vascular permeability (van Duinen et al., 2017). To evaluate the functionality and relevance of the HCAEC microvessel model, we developed an assay to quantify (i) the adhesion of monocytic cells under flow to the lumen of HCAEC microvessels as a functional readout and (ii) the intercellular adhesion molecule 1 (ICAM1; also known as CD54) protein in HCAECs as a molecular readout for adhesion (Fig. 1, Step 2). We used tumor necrosis factor $\alpha(\mathrm{TNF} \alpha)$, a prototypical inflammatory stimulus known to trigger monocyte-endothelium adhesion, and also investigated concentration-dependent gene expression changes in HCAEC microvessels using transcriptomics. As a real-case application of our $3 \mathrm{D}$ vasculature-on-a-chip model, we assessed the comparative impact of THS 2.2 aerosol and 3R4F cigarette smoke in the form of conditioned medium on the adhesion of monocytic cells to HCAEC microvessels, a key event in atherogenesis (Fig. 1, Step 3 ). After exposure to conditioned medium, glutathione (GSH) content was also measured to quantify oxidative stress promoted in endothelial microvessels similarly to smokers' blood, which acquires oxidative properties over time and may contribute to endothelial dysfunction (Fratta Pasini et al., 2012).

\section{Material and methods}

\subsection{Cell culture}

Primary HCAECs (PromoCell 12221; Heidelberg, Germany) were cultured in pre-coated tissue culture flasks (Nunc Easyflask; Roskilde, Denmark) with MV2 endothelial cell growth medium (PromoCell C-22-22) containing 5\% fetal bovine serum (FBS, Gibco 16140-071; Waltham, MA, USA) and 1\% penicillin/streptomycin (Sigma-Aldrich P4333; St. Louis, MO, USA). Mono-Mac-6 (MM6) cells (DSMZ, ACC-No.124; Braunschweig, Germany) were maintained in Roswell Park Memorial Institute (RPMI) 1640 medium (Sigma-Aldrich R0883) containing $10 \%$ FBS and $1 \%$ penicillin/streptomycin.

\subsection{OrganoPlate ${ }^{\circledR}$ culture}

We used two-lane OrganoPlates ${ }^{\circledR}$ with $400 \mu \mathrm{m} \times 220 \mu \mathrm{m}(\mathrm{w} \times \mathrm{h})$ channels (MIMETAS 9603-400B, Leiden, The Netherlands). Gel and perfusion channel lengths were $9 \mathrm{~mm}$ and $12.2 \mathrm{~mm}$, respectively. Before seeding, $50 \mu \mathrm{L}$ of Hank's Balanced Salt Solution (HBSS) were dispensed into the observation window to prevent evaporation and enhance optical clarity. $2 \mu \mathrm{L}$ of gel composed of $4 \mathrm{mg} / \mathrm{mL}$ collagen I (Cultrex rat collagen 1, $5 \mathrm{mg} / \mathrm{mL}$; Trevi- gen, Gaithersburg, MD, USA), 100 mM HEPES (Gibco 15630$056)$ and $3.7 \mathrm{mg} / \mathrm{mL} \mathrm{Na}_{2} \mathrm{HCO}_{3}$ (Sigma-Aldrich S5761) were dispensed in the gel inlet and incubated for $30 \mathrm{~min}$ at $37^{\circ} \mathrm{C}$ to allow gelation of the extracellular matrix (ECM). HCAECs at passage 3 were trypsinized using $0.025 \%$ trypsin in phosphate-buffered saline (PBS)/ethylenediaminetetraacetic acid (Lonza CC-5012; Basel, Switzerland) and resuspended in the appropriate volume $\left(10^{6}\right.$ cells $\left./ \mathrm{mL}\right)$ in MV2 medium. $2 \mu \mathrm{L}$ of cell solution were dispensed in the perfusion channel inlet of the OrganoPlate ${ }^{\circledR}$. After addition of the cells, $50 \mu \mathrm{L}$ of MV2 medium were dispensed in the perfusion inlet to prevent dehydration of the cell suspension. The plate was incubated on its side for $2 \mathrm{~h}$ to allow the cells to attach to the ECM. Subsequently, $50 \mu \mathrm{L}$ of MV2 medium were dispensed in the perfusion outlet, and the plate was placed in the incubator $\left(37^{\circ} \mathrm{C}, 5 \% \mathrm{CO}_{2}\right)$ on a rocking platform (4-min intervals at an angle of $7^{\circ}$ ), resulting in a bidirectional flow. For the culture optimization step, we used $9.1 \mathrm{mg} / \mathrm{mL}$ of Matrigel growth factor reduced basement membrane matrix (Corning 356231; Corning, NY, USA) and endothelial medium from Cell Biologics (H1168; Chicago, IL, USA). ECM and medium were mixed at a 1:1 (v/v) ratio. The medium was changed 3 times per week.

\subsection{Barrier integrity assay}

Medium in the perfusion channel was replaced by MV2 medium containing $0.5 \mathrm{mg} / \mathrm{mL} 4.4$ or $20 \mathrm{kDa}$ fluorescein isothiocyanate (FITC)-dextran (Sigma-Aldrich FD20S) and $0.5 \mathrm{mg} / \mathrm{mL}$ $155 \mathrm{kDa}$ tetramethylrhodamine isothiocyanate (TRITC)-dextran (Sigma-Aldrich T1287) (40 $\mu \mathrm{L}$ on perfusion inlet, $30 \mu \mathrm{L}$ on perfusion outlet). Ten microliters of MV2 medium were dispensed on the gel inlet. Leakage of the fluorescent probe from the lumen of the endothelial microvessel into the ECM compartment was captured using an ImageXpress XLS Micro HCI system (2-min intervals, 14 min total; Molecular Devices, San Jose, CA, USA). The ratio between the fluorescent signal in the perfusion channel and the ECM channel was analyzed using Fiji (ImageJ, NIH, Bethesda, MD, USA). The permeability of the membranes was analyzed by measuring the number of molecules that leaked though the membrane into the adjacent gel lane over time. From these measurements, the apparent permeability index $\left(\mathrm{P}_{\mathrm{app}}\right.$ : initial flux of a compound through a membrane, normalized by membrane surface area and donor concentration) was calculated using the following formula:

$$
P_{\text {app }}=\frac{\Delta C_{\text {receiver }} \times V_{\text {receiver }}}{\Delta \mathrm{t} \times A_{\text {barrier }} \times C_{\text {donor }}}\left(\frac{\mathrm{cm}}{\mathrm{s}}\right)
$$

where $\Delta C_{\text {receiver }}$ is the measured normalized intensity difference of the ECM to the donor channel at $t_{0 \min }$ and $t_{10 \text { min }}, V_{\text {receiver }}$ is the volume of the measured region in the ECM channel, $\Delta_{t}$ is the time difference, $A_{\text {barrier }}$ is the surface of the ECM interface with the medium channel, and $C_{d o n o r}$ is the donor concentration of the dextran dye $(0.5 \mathrm{mg} / \mathrm{mL})$.

\subsection{Monocyte-endothelial microvessel adhesion assay under flow}

MM6 monocytes (passage 12) were harvested and suspended at a density of $10^{5}$ cells $/ \mathrm{mL}$ in RPMI medium containing $0.5 \mu \mathrm{g} / \mathrm{mL}$ 
calcein AM (Life Technologies C3099; Carlsbad, CA, USA) for $15 \mathrm{~min}$ at $37^{\circ} \mathrm{C}$. The monocytes were spun down at $200 \times \mathrm{g}$ for $5 \mathrm{~min}$, the calcein AM-containing medium was aspirated, and the cells were resuspended at a density of $10^{5}$ cells $/ \mathrm{mL}$ in MV2 medium. Simultaneously, the nuclei of the endothelial microvessels were stained with Hoechst 33342 (1:2,000, Thermo Fisher Scientific H3570; Waltham, MA, USA) for $20 \mathrm{~min}$ at $37^{\circ} \mathrm{C}$. After washing the endothelial tube for 5 min with MV2 medium, the monocyte suspension was added to the perfusion channel and incubated at $37^{\circ} \mathrm{C}$ and $5 \% \mathrm{CO}_{2}$ for $15 \mathrm{~min}$ on a rocking platform (8-min intervals at a $7^{\circ}$ angle). After washing twice with HBSS for $5 \mathrm{~min}$, the endothelial vessel was imaged using an ImageXpress XLS-C HCI system. The number of endothelial nuclei in the microvessels was extracted using an approach based on morphological shape filtering using built-in tools available in Fiji (version 2) / ImageJ (1.52e build) (Haralick et al., 1987). Endothelial cell nuclei were extracted by removing the background signal via a Rolling Ball method (Sternberg, 1983) in the blue Hoechst channel. After the remaining signal was thresholded to highlight the nuclei, particle detection was performed to count the number of nuclei. The number of adhering monocytes was extracted using methods based on intensity thresholding and particle detection (Lee et al., 1990) in the green calcein AM channel. The method used was nearly identical to the method described for obtaining the number of endothelial nuclei. The expected minimum size for a monocyte was adjusted, because monocytes are larger than nuclei (5-9 $\mu \mathrm{m}$ for nuclei, more than $10 \mu \mathrm{m}$ for monocytes). The ratio of monocytes to endothelial cells was subsequently calculated and used as a standardized expression of monocyte adhesion to the endothelial microvessel.

\subsection{Immunohistochemistry}

Endothelial microvessels were fixed using 3.7\% formaldehyde (Sigma-Aldrich 252549) in HBSS (Sigma-Aldrich H6648) for $20 \mathrm{~min}$, washed twice with HBSS for $5 \mathrm{~min}$, and permeabilized with $0.3 \%$ Triton X-100 (Sigma-Aldrich T8787) in HBSS for $10 \mathrm{~min}$. After washing with 4\% FBS in HBSS for $5 \mathrm{~min}$, the HCAECs were incubated with a blocking buffer (2\% FBS, 2\% bovine serum albumin, Sigma-Aldrich A2153) and 0.1\% Tween 20 (Sigma-Aldrich P9416) in HBSS for 45 min. After blocking, cells were incubated for 90 min with a primary antibody solution, washed 3 times with 4\% FBS in HBSS, and incubated with a secondary antibody and nuclear staining for $30 \mathrm{~min}$. All steps were performed at room temperature. The primary antibodies used were mouse anti-human antibodies for ICAM1 at $5 \mu \mathrm{g} / \mathrm{mL}$ (1:100, Bio-Techne BBA3; Minneapolis, MN, USA), CD31 at $10 \mu \mathrm{g} / \mathrm{mL}$ (Dako M0823), and rabbit anti-human antibodies for VE-cadherin at $1 \mu \mathrm{g} / \mathrm{mL}$ (Abcam, Ab33168; Cambridge, UK). The secondary antibodies were goat anti-mouse Alexa Fluor 488 at $8 \mu \mathrm{g} / \mathrm{mL}$ (Invitrogen A11001; Carlsbad, CA, USA) and goat anti-rabbit Alexa Fluor 488 at $8 \mu \mathrm{g} / \mathrm{mL}$ (Invitrogen A11008). For nuclear staining, $5 \mu \mathrm{g} / \mathrm{mL}$ Hoechst 33342 (Thermo Fisher Scientific H3570) was used.

Images were captured using an ImageXpress XLS-C HCI system. For quantification of the immunofluorescent staining, z-series were captured and the sum projection was saved. Quantifi- cation was performed by calculating the mean intensity of the captured image in Fiji divided by the number of observed nuclei.

\subsection{Transcriptomic analysis of endothelial microvessels stimulated by TNFa}

Microvessel lysis

HCAEC microvessels were lysed by applying $40 \mu \mathrm{L}$ of lysis buffer (consisting of 1\% beta-mercaptoethanol, Sigma-Aldrich M6250, in Buffer RLT, Qiagen 79216; Hilden, Germany) to the perfusion inlet and $10 \mu \mathrm{L}$ lysis buffer to the perfusion outlet, creating a flow through the microfluidic channel. After $30 \mathrm{sec}$, the lysate was collected in a polymerase chain reaction clean tube (VWR 211-2120; Radnor, PA, USA). Seven microfluidic chips were pooled into a total volume of $240 \mu \mathrm{L}$ lysis buffer and stored at $-80^{\circ} \mathrm{C}$ for transcriptomic analysis.

\section{RNA extraction and profiling}

Samples from pooled HCAEC microvessel lysates from 4 independent experiments were randomized, and RNA was extracted on a QIAcube robot (batch of 12 samples) using the RNeasy Micro Kit (Qiagen 74004) and then stored at $-80^{\circ} \mathrm{C}$. Purified RNA was quantified using a Nanodrop ND-1000 (Thermo Fisher Scientific). The RNA integrity number was determined using an Agilent 2100 Bioanalyzer with the Agilent RNA 6000 Pico Kit (Agilent Technologies 5067-1513; Santa Clara, CA, USA); RNA integrity number values ranged from 6.8 to 10 (mean: 9.58). RNA was processed in a 96-chip plate (same batch) and required $50 \mathrm{ng}$ of total RNA on a fully automated Biomek FXp robot (Beckman Coulter, Brea, CA, USA) with the NuGEN Ovation RNA amplification system V2 protocol (3100-A01; San Carlos, CA, USA). Single-primer isothermal amplification was followed by fragmentation and biotinylation of the cDNA. Hybridization was performed overnight $(16 \mathrm{~h})$ at $60 \mathrm{rpm}$ in a $45^{\circ} \mathrm{C}$ GeneChip ${ }^{\circledR}$ Hybridization Oven 645 (Affymetrix, Santa Clara, CA, USA) on a GeneChip Human Genome U133 Plus 2.0 Array (Affymetrix), which measures the expression of more than 47,000 transcripts. The sequences from which these probe sets were derived were selected from GenBank ${ }^{\mathrm{TM}}$, dbEST, and RefSeq sequence databases. Arrays were washed and stained on a GeneChip ${ }^{\circledR}$ Fluidics Station FS450 DX (Affymetrix) using the Affymetrix GeneChip ${ }^{\mathrm{TM}}$ Command Console Software (AGCC software version 3.2, protocol FS450_0004).

\section{Transcriptomic data processing and pairwise comparisons}

Summarization and normalization of probes in the raw data (CEL files with access ID E-MTAB-7555 are available in the ArrayExpress public repository database) were performed using Entrez-based probe annotation HGU133Plus2_Hs_ENTREZG cdf version 16.0.0 (Dai et al., 2005) and frozen robust microarray analysis (fRMA) (McCall et al., 2010), respectively. The normalization vector HGU133Plus2_fRMAvecs version 1.3.0 was used with the R package fRMA version 1.18.0. Quality controls, including log intensities, normalized unscaled standard error, relative log expression, median absolute value of relative log expression, and pseudo-images and raw image plots, were performed using the affyPLM package version 1.42.0 (Bioconductor suite) 
(Bolstad, 2005). After quality control, pairwise comparisons at the gene level, called systems response profiles (SRP), were computed by comparing each concentration treatment with its respective vehicle control (VC) using the Bioconductor Limma $\mathrm{R}$ package version 3.22.1 (Smyth, 2004). No gene was filtered out at any step of the computational analysis. Genes with a false discovery rate (FDR) below 0.05 ( $p$-value adjustment using the Benjamini and Hochberg method) were considered differentially expressed (Benjamini and Hochberg, 1995). For biological interpretation, SRPs including all genes $(18,604)$ were analyzed using $p$-value threshold-free computational approaches (gene set enrichment analysis and network perturbation amplitude (NPA) analyses described below). Most $\mathrm{R}$ packages used to conduct the data analysis were included in Bioconductor version 3.0. The $\mathrm{R}$ version used was 3.1.2.

\section{NPA analysis}

Using transcriptomic data and biological cause-and-effect network models, we applied a computational approach that quantifies a response to a stimulus to analyze network perturbation (Martin et al., 2014) in HCAEC microvessels. A network is an assembly of directed and signed causal relationships between molecular biological entities that collectively model a specific biological process in a defined context (e.g., lung or vascular). The causal relationships have been curated from the scientific literature and encoded in biological expression language (BEL) syntax. The network is composed of functional (backbone) and transcript (gene expression) layers. The backbone node-level and network-level perturbation amplitudes can be calculated by considering the measured gene expression changes and the network topology using a backward-causal reasoning algorithm. Three statistics are computed to assess the significance of an NPA score with respect to biological variation (confidence interval) and its specificity to the given two-layer network structure ( $\mathrm{O}$ and $\mathrm{K}$ statistics). A collection of biological networks representative of the main processes of cell fate, inflammation, repair, stress and proliferation has been published ${ }^{1}$ (sbv IMPROVER project team et al., 2013; Boue et al., 2015). We report here the NPA scores for the vascular inflammatory processes/endothelial cell-monocyte interaction network model in the scope of this manuscript.

\section{Principal component (PC) analysis (PCA) and pathway enrichment analysis}

Gene set enrichment analysis was conducted to identify biological pathways/processes associated with genes that drive the discrimination of concentration and exposure time of HCAEC microvessels to TNF $\alpha$. After performing PCA of the gene expression fold-change (FC) matrix, genes were ranked by their contribution to PCs that explained the maximum of variance. Gene set enrichment analysis was performed using the MSigDB C2-CP gene set collection to identify enriched gene sets representative of biological canonical pathways/processes (Subramanian et al., 2005). Gene resampling (Q1) was per- formed to generate the null hypothesis distribution and compute the significance associated with each gene set (Ackermann and Strimmer, 2009).

\subsection{Preparation of THS 2.2- and 3R4F-conditioned media from $\mathbf{M} \mathbf{M 6}$ cells}

Generation of fresh aqueous extracts from THS 2.2 aerosol or $3 R 4 F$ smoke

Mainstream smoke from the 3R4F reference cigarette (Kentucky Tobacco Research Center, University of Kentucky, Lexington, KY, USA) was generated on a 20-port rotary Borgwaldt smoking machine (Hamburg, Germany) according to the Health Canada Intense protocol: $55 \mathrm{~mL}$ puff volume, 30-s puff interval, with all ventilation holes blocked (Government of Canada, 2000). The smoke generated from 6 reference cigarettes was bubbled through $36 \mathrm{~mL}$ of ice-cold RPMI 1640 culture medium to trap the water-soluble fraction, resulting in a stock solution concentration of approximately $1.8 \mathrm{puffs} / \mathrm{mL}$, corresponding to about 10.7 puffs per cigarette, on average. Mainstream aerosol from the candidate MRTP THS 2.2, developed by Philip Morris International, was produced using a pre-defined puff count of 12 puffs per stick on a 30-port rotary aerosol generator (type SM 2000 P1), according to the Health Canada protocol. The aerosol was bubbled into ice-cold RPMI 1640 culture medium to trap the water-soluble fraction $(10$ HeatSticks $/ 40 \mathrm{~mL}$; stock solution concentration: three puffs $/ \mathrm{mL}$ ). Previous analysis of aqueous extracts showed that the concentrations of carbonyls were significantly lower in THS 2.2 aqueous extract compared with 3R4F aqueous extract, while nicotine remained at comparable concentrations in both extracts (Poussin et al., 2016, 2018).

\section{Exposure of MM6 cells to fresh THS 2.2 or $3 R 4 F$ aqueous extracts to generate conditioned media}

An MM6 cell suspension was adjusted to $2 \times 10^{6}$ cells $/ \mathrm{mL}$ in RPMI 1640 medium supplemented with $10 \% \mathrm{FBS}$, and $0.5 \mathrm{~mL} /$ well was seeded in a 24-well plate. MM6 cells were exposed to various concentrations of 3R4F/THS 2.2 aqueous extract or culture medium $/ 10 \%$ FBS (VC) for $2 \mathrm{~h}$. Culture supernatants, namely conditioned media, were collected and stored at $-80^{\circ} \mathrm{C}$ for the adhesion assay.

\subsection{Treatment of endothelial microvessels with TNFa or 3R4F/THS 2.2-conditioned medium prior to adhesion assay and marker staining}

On the $4^{\text {th }}$ day after seeding, endothelial microvessels were treated with TNFa (ImmunoTools 11343015; Friesoythe, Germany) as a positive control for inflammation, with L-buthionine-sulfoximine (25 $\mu \mathrm{M}$, Sigma-Aldrich B2515) or ethacrynic acid ( $25 \mu \mathrm{M}$, Sigma-Aldrich E4754) as positive controls for GSH depletion, or with 3R4F- or THS 2.2-conditioned medium for 4 or $16 \mathrm{~h}$. The $4 \mathrm{~h}$ time point was used for comparison with previous results obtained in a static 2D endothelial cell model (Poussin et al., 2015, 2016). Adhesion proteins, e.g., SELE, are already max-

\footnotetext{
1 http://causalbionet.com
} 


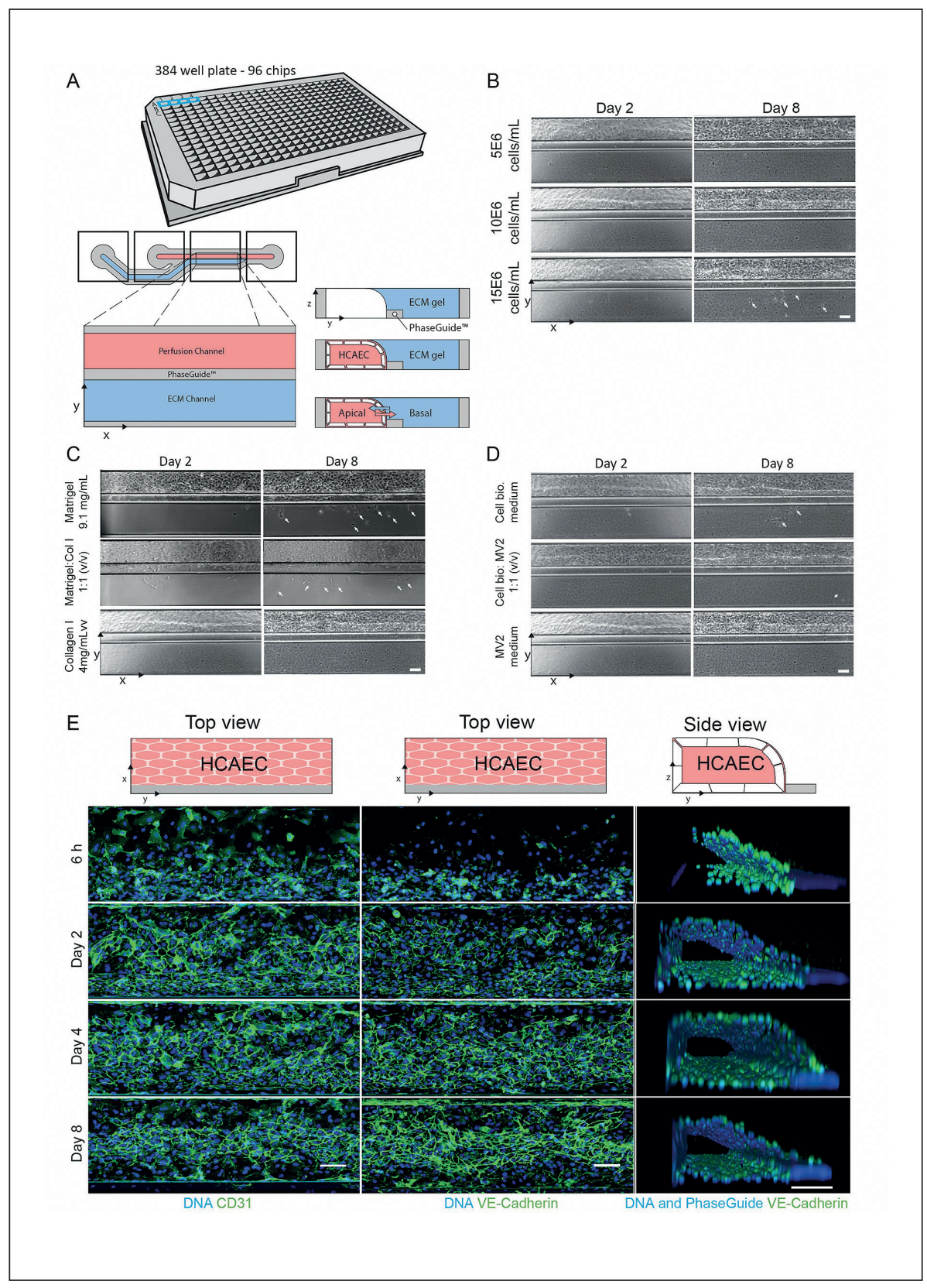

Fig. 2: 3D endothelial microvessel culture formation and optimization (A) Schematic overview of the OrganoPlate ${ }^{\circledR}$. A gel channel (blue) holds an ECM in place through the PhaseGuide's pressure barrier function. Endothelial cells are seeded in the adjacent medium perfusion channel (red). Upon the addition of perfusion flow, a tubular structure is formed in the medium channel. (B-D) 3D culture optimization of primary human coronary artery endothelial cells on the OrganoPlate ${ }^{\circledR}$ platform. The platform was optimized for cell seeding density (B), ECM composition (C), and medium composition (D) to select the optimal cell culture conditions. Phase contrast pictures were taken 2 and 8 days after seeding, and the optimal conditions (barrier formation and no invasion of the ECM) were selected for subsequent experiments. White arrows indicate invasion of cells into the ECM on day 8 . (E) Maximum projection and 3D reconstruction of a confocal z-stack of the cells in the tissue chips using the optimized seeding conditions $\left(10^{6}\right.$ cells/ $\mathrm{mL}$, collagen I at $4 \mathrm{mg} / \mathrm{mL} \mathrm{ECM}$, and MV2 medium). HCAEC microvessels were fixed $6 \mathrm{~h}$ and 2,4 and 8 days after seeding and stained for DNA and the adherens junction markers CD-31 and VE-cadherin. The maximum projections and $3 \mathrm{D}$ reconstruction show barrier formation and stable tubular morphology with a lumen 2 to 8 days after seeding. Scale bar $=100 \mu \mathrm{m}$.

imally expressed after $4 \mathrm{~h}$ in endothelial cells, and their expression may be transient and decrease at later time points, while the expression of other adhesion proteins, such as vascular cellular adhesion molecule 1, is maintained or only upregulated at later time points (i.e., $16 \mathrm{~h}$ ) (Granger and Senchenkova, 2010). At the $4 \mathrm{~h}$ time point, primary effects of a stimulus can be investigated, while at later time points, secondary activation occurs via feedback loops. At the end of the treatment, endothelial microvessels were either fixed for specific marker immunohistochemistry staining, lysed for transcriptomic analysis (only for TNF $\alpha$ treatment), or directly nuclear stained and used for the adhesion assay under flow.

\subsection{Measurement of GSH content}

Endothelial microvessels were incubated with MV2 medium containing $0.125 \mathrm{mg} / \mathrm{mL}$ monochlorobimane (Sigma-Aldrich 69899) and $5 \mu \mathrm{M}$ DRAQ5 (Abcam ab108710) for $30 \mathrm{~min}$ at $37^{\circ} \mathrm{C}$ and $5 \% \mathrm{CO}_{2}$ on a rocking platform (8-min intervals at a $7^{\circ}$ angle). After washing with HBSS, the z-series were captured on an ImageXpress XLS-C HCI system. Quantification of the intensity of the fluorescent signal was performed in Fiji. Sum projections were acquired from the z-series, and the mean intensity was calculated after subtracting the background. Subsequently, the intensity per counted nucleus was calculated. 


\subsection{Water-soluble tetrazolium salt (WST-8) viability assay}

Medium in endothelial microvessels was replaced with MV2 medium containing Cell Counting Kit- 8 solution (1:11 dilution, Sigma-Aldrich 96992) and incubated at $37^{\circ} \mathrm{C}$ and $5 \% \mathrm{CO}_{2}$ for 30 min on a rocking platform (8-min intervals at a $7^{\circ}$ angle). After incubation, the absorbance was measured at $450 \mathrm{~nm}$ on a plate reader (Multiskan FC, Thermo Fisher Scientific). The measurements of the gel inlet, perfusion inlet and outlet, and observation window were adjusted for volume differences and combined after background subtraction of the positive cell-free control. Analysis was performed in Microsoft Excel (Redmond, WA, USA). Data was normalized to the negative control (MV2 $\rightarrow$ MV2 0 pg/ $\mathrm{mL} \mathrm{TNF} \alpha$ ) for the "starvation experiment" and the conditioned medium vehicle control (corresponding to supernatant of MM6 cells exposed to 0 puffs $/ \mathrm{mL}$ smoke/aerosol aqueous extract) for the "conditioned medium exposure experiment."

\subsection{Statistical analysis}

Statistical analysis was conducted in GraphPad Prism (La Jolla, CA, USA). The mean values for all within-OrganoPlate ${ }^{\circledR}$ chip replicates were calculated and normalized to the control (ratio value/control). Statistical analysis was performed with a one- or two-way analysis of variance (ANOVA) followed by post-hoc Dunnett's pairwise comparisons. The number of replicates for chips within an OrganoPlate ${ }^{\circledR}(\mathrm{n})$ and independent experiments $(\mathrm{N})$ is indicated in the figure legends for each experiment.

Datasets and further detail on the protocols and additional data visualizations are available on the INTERVALS platform ${ }^{2}$.

\section{Results}

\subsection{Development and characterization of a perfused human coronary artery endothelial microvessel}

\subsubsection{Development and optimization of conditions for the formation of endothelial microvessels}

Endothelial microvessels were cultured in the two-lane $400 \mu \mathrm{m}$ OrganoPlate $^{\circledR}$ (Fig. 2A). HCAEC vessel formation was optimized for seeding density (Fig. 2B), ECM (Fig. 2C) and medium composition (Fig. 2D). Rapid vessel formation and vessel stability for at least 8 days without ingrowth of HCAECs into the ECM were achieved with a seeding density of $10^{6}$ cells $/ \mathrm{mL}$ together with collagen I $4 \mathrm{mg} / \mathrm{mL}$ ECM cultured in MV2 medium. All further experiments were conducted using this optimized seeding protocol. To show stable vessel formation and endothelial marker expression, endothelial microvessels were fixed at different time points; 3D reconstructions show the reproducible formation of a complete vessel after 2 days. The vessel remained stable with regards to the expression of CD31 (PECAM-1), VE-cadherin at the junctions (Fig. 2E), and low expression of the apoptosis markers caspase $3 / 7$ (Fig. S1 ${ }^{3}$ ) for at least 8 days (Fig. 2E).

\subsubsection{Barrier integrity increases in the course of endothelial microvessel formation and remains stable up to 8 days}

To assess barrier formation in the HCAEC microvessels, a barrier integrity assay was performed (Fig. 3A). Briefly, a fluorescent reporter molecule was perfused through the lumen of the vessel (perfusion channel) and followed over time to assess the diffusion of the reporter into the gel channel. The fluorescence intensity of the medium and gel channel was measured to calculate the apparent permeability coefficient. The barrier integrity assay was performed with three sizes of dextran molecules $(4.4,20$ and $155 \mathrm{kD}$ ). Representative images of microvessels at different time points are shown in Figure 3B. Figure 3C shows barrier formation over time. One day after introducing the HCAECs into the microfluidic channels, the diffusion of both 4.4-kD and $20-\mathrm{kD}$ dextran through the barrier began to decrease compared with diffusion in controls without cells, indicative of the formation of an endothelial cell barrier that remained stable for up to 8 days.

\subsubsection{Establishment of a monocyte-endothelial microvessel adhesion model under flow}

To test the endothelial microvessel model for monocyte attachment, fluorescently labeled MM6 monocytes were introduced into the endothelial microvessels after vessel stimulation with increasing concentrations of TNF $\alpha$ for 4 or $16 \mathrm{~h}$ under flow. After 15 min of incubation under perfusion flow, non-attached monocytes were washed away, and the adherent monocytes were quantified. Representative images are shown in Figure 4A. Quantification shows a significant concentration-dependent upregulation of monocyte attachment after stimulation for 4 and $16 \mathrm{~h}$ for all tested concentrations of TNF $\alpha$ compared with attachment in the VC (Fig. 4B). At the 4-h time point, the FC of adherent monocytes reached a plateau at concentrations of 50-100 pg/mL TNF $\alpha$ (Fig. 4B). At the 16-h time point, the FC of adherent monocytes tended to decrease at the lowest tested concentrations (100 and $500 \mathrm{pg} / \mathrm{mL})$ of TNF $\alpha$, but was maintained at higher concentrations (500 and $1,000 \mathrm{pg} / \mathrm{mL})$ and increased at the highest concentration $(10,000 \mathrm{pg} / \mathrm{mL})$, compared with the FCs at the $4 \mathrm{~h}$ time point (Fig. 4B). In parallel to monocyte adhesion, ICAM1, an adhesion molecule expressed by endothelial cells to which monocytes bind (Fig. 4C), was quantified in endothelial microvessels. Stimulation of the endothelial microvessels with TNF $\alpha$ significantly upregulated ICAM1 protein levels in HCAECs in a concentration-dependent manner compared with levels in the VC. Representative images are shown in Figure 4D, and quantification of the immunofluorescent images is shown in Figure 4E. Longer stimulation with high concentrations of TNF $\alpha$ (more than $500 \mathrm{pg} / \mathrm{mL}$ ) resulted in higher ICAM1 levels. The profiles of concentration-dependent ICAM1 protein abundance FCs observed at 4 and $16 \mathrm{~h}$ correlated positively with those of monocyte-endothelial microvessel adhesion at the corresponding time points (Fig. 4B,E).

\footnotetext{
2 doi:10.26126/intervals.elcwt4.1

3 doi:10.14573/altex.1811301s
} 

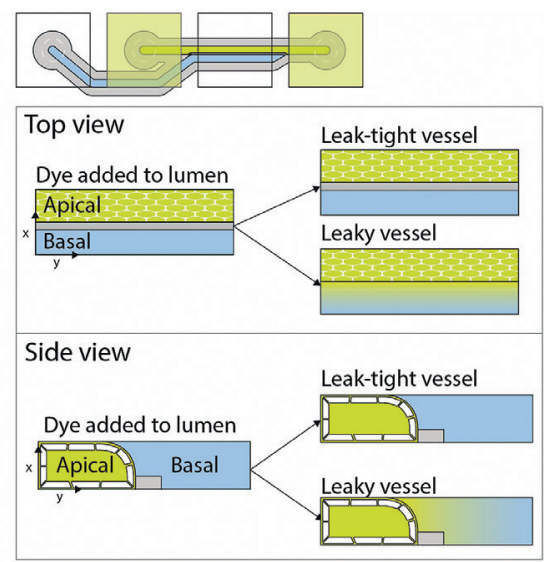

B
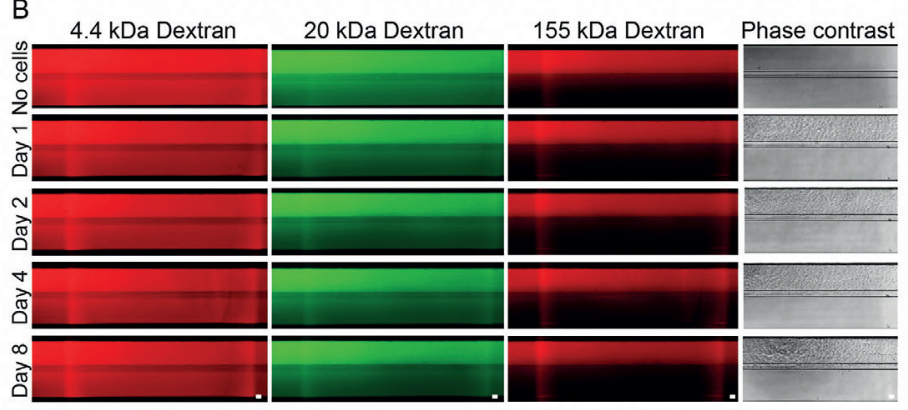

HCAEC barrier formation

C

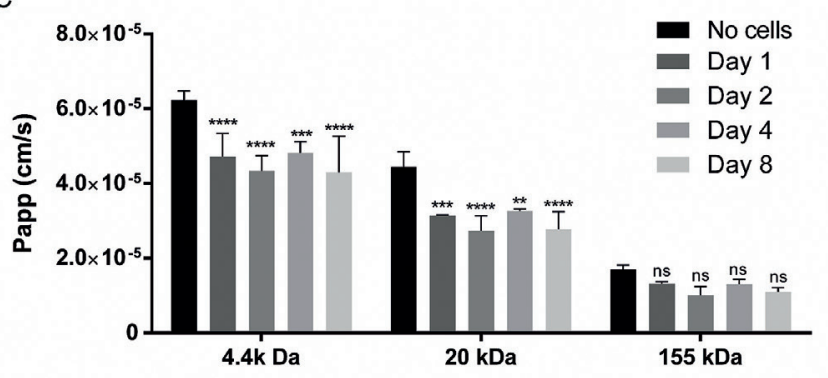

Fig. 3: Barrier formation in HCAECs

(A) Principle of the barrier integrity assay in the OrganoPlate ${ }^{\circledR}$. A fluorescent dye is added to the lumen of the vessel, and the integrity of the barrier is quantified by measuring the intensity of fluorescent dye diffusing into the adjacent gel channel.

(B) Fluorescent images show HCAEC barriers for both $4.4 \mathrm{kDa}$ FITC-dextran, 20 kDa FITC-dextran and 155 TRITCdextran 1 to 8 days after seeding. The fluorescent images were taken 14 minutes after addition of the dye. Scale bar (white color) at the bottom = $100 \mu \mathrm{m}$. (C) $\mathrm{P}_{\mathrm{app}}$ index of the HCAEC barrier over time. Plot bars represent the mean + SD $(N=3, n=4-7)$; ns, not significant; ${ }^{* *}, p<0.01 ;{ }^{* * *}, p<0.001$; $* * \star *, p<0.0001$ (two-way ANOVA).

\subsubsection{Transcriptomic analyses of HCAEC microvessels stimulated by TNFa for 4 and $16 \mathrm{~h}$}

Transcriptome analysis of HCAEC microvessels enabled the investigation of molecular changes at the mRNA transcript level following treatment with TNF $\alpha$ for 4 and $16 \mathrm{~h}$. SRPs (or contrasts) were computed by comparing each TNF $\alpha$ treatment group with its respective $\mathrm{VC}$ using linear modeling. A PCA plot of the FC matrix visualized the sources of variation in gene expression data. Scores (treatment groups) and loadings (genes contributing to the discrimination of treatment groups) were visualized on a biplot for PC1-PC2 sub-space, both PCs explaining 73.1\% of the total variance (Fig. 5A,B), and revealed time and concentration effects, with a clear separation of SRPs mostly along PC1 and
PC2. Top genes with the largest positive and negative loading values for PC1 and PC2 (Fig. 5B) showed specific FC expression patterns across concentrations and time points (Fig. 5C). For biological interpretation, all genes were ranked by their contribution to $\mathrm{PC} 1$ or $\mathrm{PC} 2$, and gene set enrichment analysis was performed using the MSigDB C2-CP gene set collection representative of canonical pathways/biological processes. Gene sets grouped by their positive and negative enrichment scores were scattered in the four quadrants of the PCA biplot. The top five significant (FDR < 0.05 for at least one PC) gene sets for each quadrant are highlighted in Figure 5D. The results show an enrichment of gene sets reflective of inflammation (e.g., KEGG_CYTOKINE_CYTOKINE_RECEPTOR_INTERACTION, PID_CD40_PATHWAY), 
A

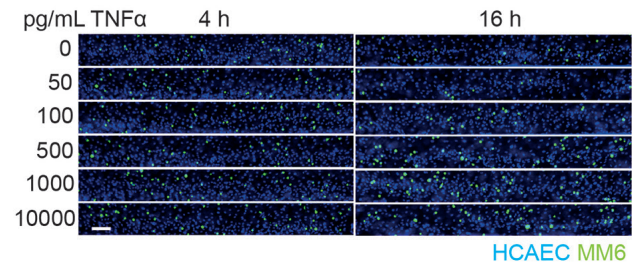

B

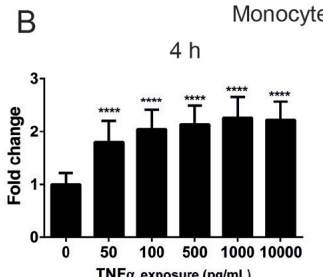
TNF $\alpha$ exposure $(\mathrm{pg} / \mathrm{mL})$

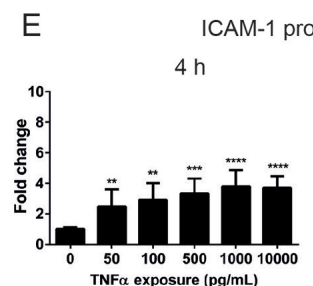

$\mathrm{TNF} \alpha$ exposure $(\mathrm{pg} / \mathrm{mL})$

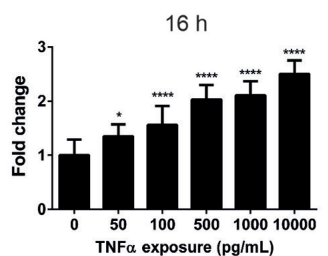

$16 \mathrm{~h}$

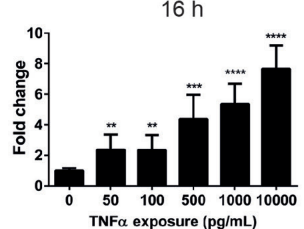

C
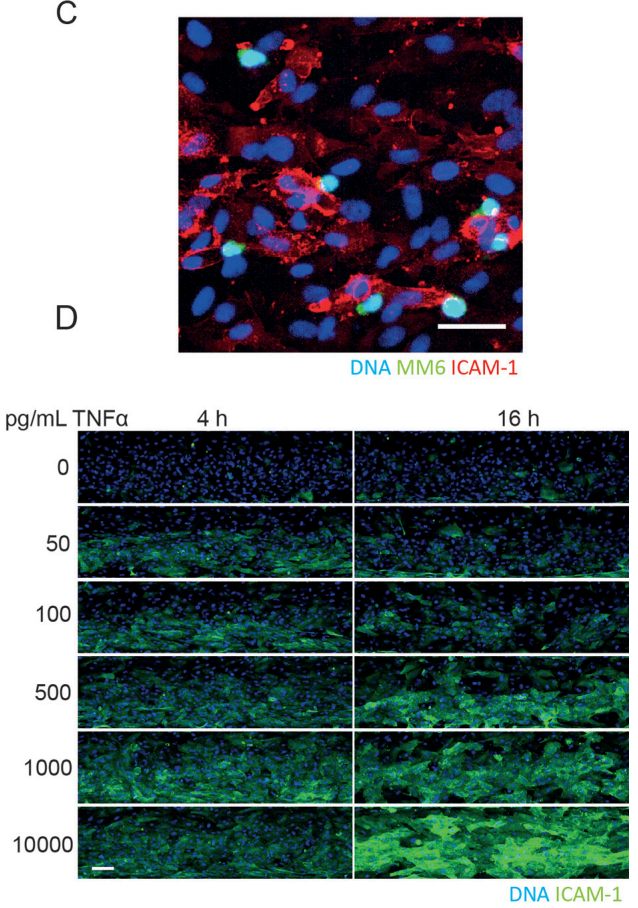

Fig. 4: Establishment of a monocyte-endothelial microvessel adhesion model under flow

(A) Monocyte adhesion after 4 or 16 h of TNFa exposure. MM6 monocytes labeled with live stain calcein AM were added under flow to the endothelial microvessel (pre-stained with the nuclear dye Hoechst 33342) for 15 minutes. After washing, fluorescent images were captured (scale bar $=50 \mu \mathrm{m}$ ) and the number of attached monocytes (green) per nucleus (blue) was calculated. (B) The plot shows the mean increase compared to the VC + SD. ${ }^{*} p<0.05$, ${ }^{* * * *} p<0.0001$ (one-way ANOVA followed by post-hoc Dunnett's pairwise comparisons) compared with VC; $\mathrm{N}=2-3, \mathrm{n}=6$. (C) High magnification of monocyte-endothelial attachment (scale bar $=50 \mu \mathrm{m})$. (D and $\mathrm{E}) \mathrm{Images}$ and quantification of the protein adhesion marker ICAM1 in the endothelial microvessel after 4 or $16 \mathrm{~h}$ of exposure to TNFa. The intensity of the immunofluorescent ICAM1 staining was measured per nucleus of HCAECs. $N=3, n=3$. The plots show the fold change relative to the VC corresponding to the condition with no TNFa (mean + SD). ${ }^{* *}, p<0.01 ;{ }^{* * *}, p<0.001 ;{ }^{* * * *}, p<0.0001$ (one-way ANOVA followed by posthoc Dunnett's pairwise comparisons).

protein post-translational modification (e.g., REACTOME_BIOSYNTHESIS_OF_THE_N_GLYCAN_PRECURSOR_DOLICHOL_LIPID_LINKED_OLIGOSACCHARIDE_LLO_AND_ TRANS̄FER TO A NA $\bar{S} C E N T$ PROTEIN), cell cycle/DNA replication (e.g., REACTOME_E2F_MEDIATED_REGULATION_OF_DNA_REPLICATION), and mitosis (e.g., PID_AURORA_B_PATHWAY, REACTOME_MITOTIC_PROMETAPHASE) pointed in the direction of the Q1, Q2, Q3, and Q4 quadrants, respectively (Fig. 5D and Tab. $\mathrm{S}^{3}$ ). The selection of differentially expressed genes $(\mathrm{FDR}<0.05)$ revealed a TNF $\alpha$ concentration-dependent increase of significantly upregulated and downregulated genes with a lesser effect at $16 \mathrm{~h}$ than at $4 \mathrm{~h}$ (Fig. 5E).

To verify consistency between molecular changes and functional readouts, we conducted a network perturbation analysis leveraging each SRP and a causal network model representative of monocyte-endothelial cell adhesion mechanisms. Figure 5F shows a TNF $\alpha$ concentration-dependent increase in the NPA simi- lar to the readout observed with the MM6 cell-HCAEC microvessel adhesion assay (Fig. 4B). Interestingly, the nodes with the larger significant amplitude scores across SRPs were "p(HGNC:ICAM1)" and "path(SDIS:monocyte_adherence)," corresponding to predicted activation of the protein ICAM1 and the adhesion of monocytes to the endothelium, respectively (Tab. S2 ${ }^{3}$ ). This result was supported by an increased expression of mRNA transcripts coding for adhesion molecules as measured by Affymetrix microarray (Fig. S2 ${ }^{3}$ ).

We then compared the response to TNF $\alpha$ in our $3 \mathrm{D}$ model and a 2D model to assess whether a similar biology was perturbed. For this purpose, we retrieved an SRP from a previous study, corresponding to the conditions of $2 \mathrm{D}$ monolayer HCAECs treated with $10 \mathrm{ng} / \mathrm{mL}$ of TNF $\alpha$ for $4 \mathrm{~h}$ and the respective VC (Poussin et al., 2018). Using the same conditions in our 3D model, a comparison of gene expression FCs and pathway-based gene set enrichment scores revealed a high consistency between the 3D and 2D models (Fig. 5G,H; Tab. S3 ${ }^{3}$ ). The correlation increased nota- 
A

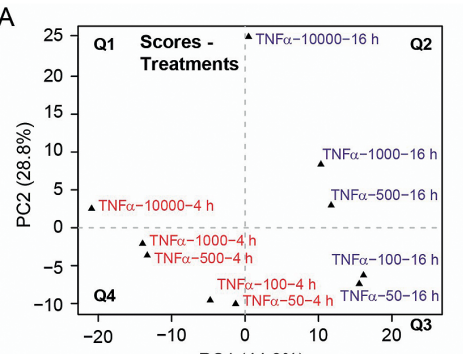

B

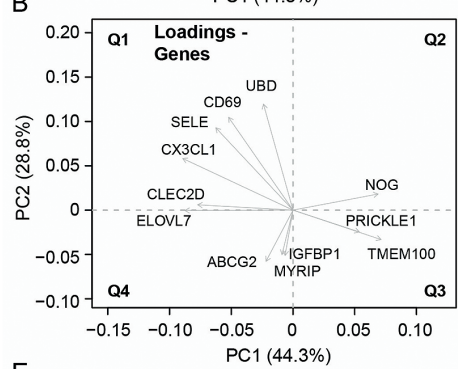

E

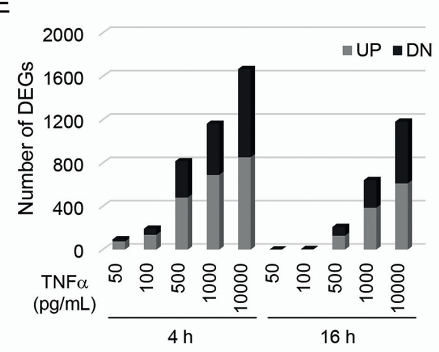

G

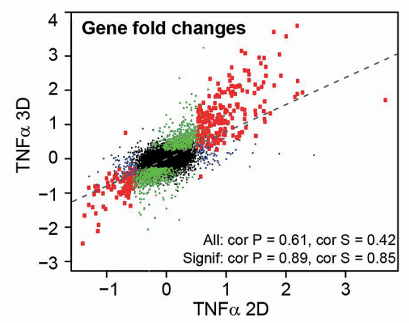

C

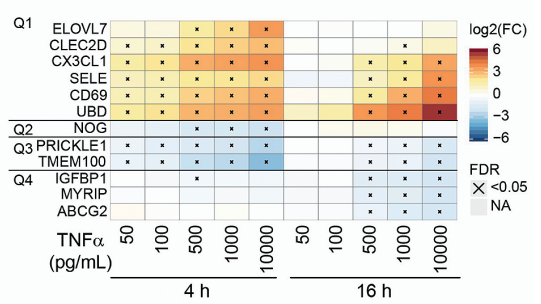

D

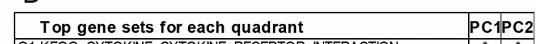

Q1 KEGG_CYTOKINE_CYTOKINE_RECEPTOR_INTERACTION

PID_CD40_PATHWAY

REACTOME_INTERFERON_GAMMA_SIGNALING

REACTOME_CYTOKINE_SIGNALING_IN_IMMUNE_SYSTEM

Q2 REACTOME_BIOSYNTHESIS OF THE_N_GLYCAN_PRECURSO

REACTOME_Q2_M_CHECKOINTS
REACTOMEACTIVATIONOF_THE_PRE_REPLICATNE_COMPLEX

REACTOME_E2F_MEDIATED_REGULATION_OF_DNA_REPLICATION

REACTOME_ACTINATION_OF_ATR_IN_RESPONSE_TO_REPLICATIO

REACTOME_G1S_SPECIFIC_TRANSCRIPTION

PIDAAURORA_BPATHWAY

PIDFOXM1-PATHWAY

PDDPLK1PATHWAY PROMTAPHASE

F $\quad 0_{3} \quad \begin{gathered}\text { VIP/Endothelial Cell-monocyte } \\ \text { Interaction }\end{gathered}$

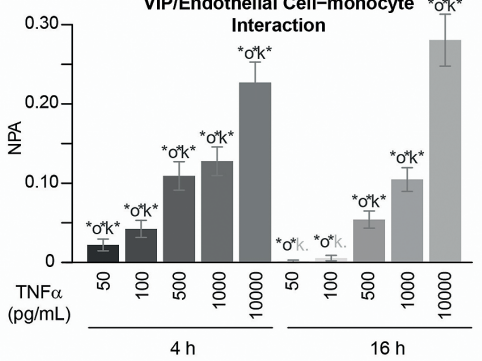

$\mathrm{H}$

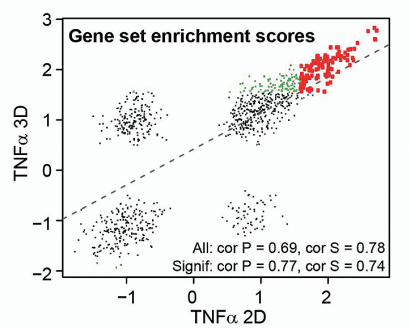

Fig. 5: Transcriptomic analysis of HCAEC microvessels at day 4 treated with various concentrations of TNFa for 4 and $16 \mathrm{~h}$

PC1-PC2 sub-space scatterplots of (A) treatment group scores and (B) gene loadings following principal component analysis of FC gene expression matrix. (C) Heatmap of expression FCs (in log2 scale) of the top six genes per principal component contributing the most to the separation of treatment groups. (D) Top gene sets associated with genes pointed in the direction of the four quadrants of the PC1-PC2 sub-space scatterplot. Gene sets were ranked by their average FDRs associated with PC1 and PC2 enrichment scores, and the top five gene sets (if more than five gene sets appeared on the list) were extracted and displayed. The full list of significant gene sets is provided in Tab. $S 1^{3}$. (E) Barplot of differentially expressed genes (FDR < 0.05) for all systems response profiles. (F) Barplot of NPA scores for microvessel systems response profiles graphed using the vascular inflammatory processes/endothelial cell-monocyte interaction network model. Scores are shown with their confidence intervals accounting for experimental variation. A network is considered perturbed if, in addition to the significance of the NPA score with respect to the experimental variation, the two companion statistics $(\mathrm{O}$ and $\mathrm{K})$ derived to inform the specificity of the NPA score with respect to the biology described in the network are significant. ${ }^{*} \mathrm{O}$ and $\mathrm{K}$ statistic $p$-values below 0.05 and NPA significance with respect to the experimental variation. $p$-values between 0.05 and 0.1 for "O" and "K" (in grey). (G) Scatterplot of gene expression FCs in log2 2D versus 3D models (TNFa, $10 \mathrm{ng} / \mathrm{mL}, 4 \mathrm{~h}$ exposure). (H) Scatterplot of gene set enrichment scores for 2D and 3D models (TNFa, $10 \mathrm{ng} / \mathrm{mL}, 4 \mathrm{~h}$ exposure). For panels $\mathrm{G}$ and $\mathrm{H}$, data points highlighted in red, green, and blue correspond to genes or gene sets that are significant in both 3D and 2D models, in the 3D model only, and in the 2D model only, respectively. The dotted line corresponds to the regression line when considering all genes or gene sets for the linear model. All Spearman and Pearson correlation coefficients were computed using all genes/gene sets; significant genes/gene sets were highly significant $(p<0.0001)$. The full list of significant gene sets is provided in Tab. S3 ${ }^{3}$. For the 3D model, $N=4$ independent experiments, $n=7$ microvessels pooled in one lysate. cor $P$, Pearson's correlation coefficient; cor S, Spearman's correlation coefficient; DN, downregulated; Signif, significant; UP, upregulated. 


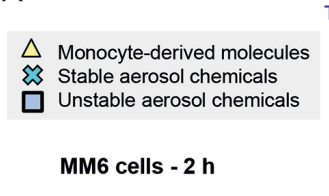

THS 2.2 or $3 \mathrm{R} 4 \mathrm{~F}$ Aqueous extract freshly prepared
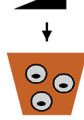

$\downarrow$

Collect and freeze conditioned medium HCAEC microvessel -4 or $16 \mathrm{~h} \downarrow \mathbb{\Xi}$

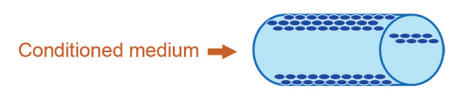

Adhesion Assay \& Marker Immunohistochemistry

C

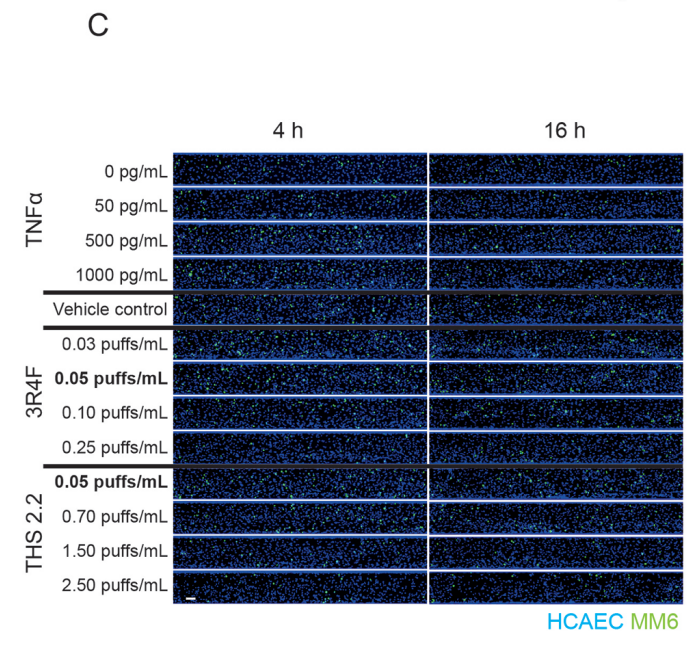

B

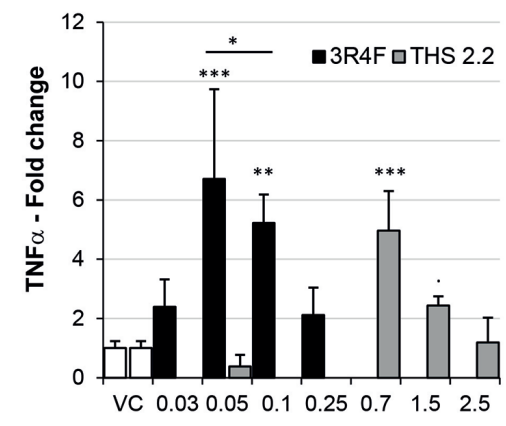

Aqueous extract (puff/mL)

D

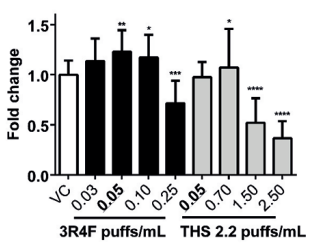

$16 \mathrm{~h}$

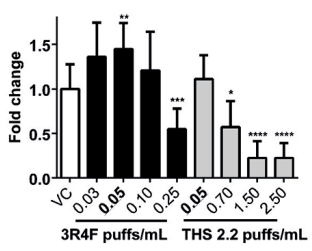

$T N F \alpha$

$4 \mathrm{~h}$

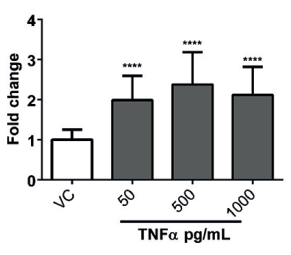

$16 \mathrm{~h}$

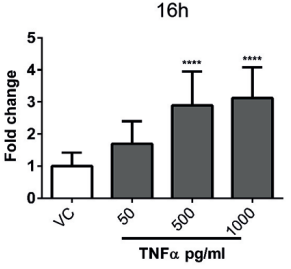

Fig. 6: 3D human microvessel-on-a-chip application for assessment of THS 2.2: monocyte-to-endothelial microvessel adhesion under flow

(A) Scheme of experimental design. (B) TNFa release in conditioned medium following a 2-h exposure of MM6 cells to freshly generated aqueous extract from 3R4F cigarette smoke or THS 2.2 aerosol. $N=4, n=2$. (C) Representative images of monocyte attachment to 4 day-HCAEC microvessels after exposure to TNFa or conditioned medium for 4 and $16 \mathrm{~h}$. (D) Quantification of monocyte adhesion after conditioned medium exposure. MM6 attachment to the endothelial microvessels was measured 4 and $16 \mathrm{~h}$ after addition of the conditioned medium. Attachment was quantified as the number of MM6 cells attached per 100 HCAECs and normalized for the VC corresponding to conditioned medium without 3R4F smoke/THS2.2 aerosol aqueous extracts. $\mathrm{N}=4, \mathrm{n}=4-6 .{ }^{*}, p<0.05 ;{ }^{* *}, p<0.01 ;{ }^{* * *}, p<0.001 ;{ }^{* * * *}$, $p<0.0001$ compared with attachment in VC (one-way ANOVA followed by post-hoc Dunnett's pairwise comparisons).

bly when selecting significant genes $(\mathrm{FDR}<0.05)$ and gene sets (FDR $<0.10)$, highlighted as red dots on the scatter plots (Fig. $5 \mathrm{G}, \mathrm{H})$. A subset of gene sets show inversely correlated enrichment scores, which were not significant (Fig. 5H).

\subsection{Model application for product toxicological assessment: use case with a heat-not-burn tobacco product compared with a reference product}

As an application of the established monocyte-endothelial microvessel adhesion model, we assessed the impact of a candidate MRTP, THS 2.2, compared with that of the 3R4F reference cigarette on the adhesion of monocytic cells to endothelial microvessels, a key step in the initiation of atherogenesis. In a previously established 2D and static adhesion assay, HCAECs were exposed to conditioned medium generated from human monocytic MM6 cells treated with various concentrations of aqueous extract of 3R4F smoke or THS 2.2 aerosol (Poussin et al., 2016). We used the same type of endothelial microvessel exposure.

\subsubsection{Reduced effect of THS 2.2 aqueous extract compared with that of $3 R 4 F$ aqueous extract on TNFa release by $\mathrm{MM} 6$ cells}

Cigarette smoke-derived chemicals that are water-soluble can interfere with serum proteins present in the culture medium, potentially reducing the effect of the smoke/aerosol aqueous extract (Grigoryan et al., 2016). To limit this, conditioned medium is 

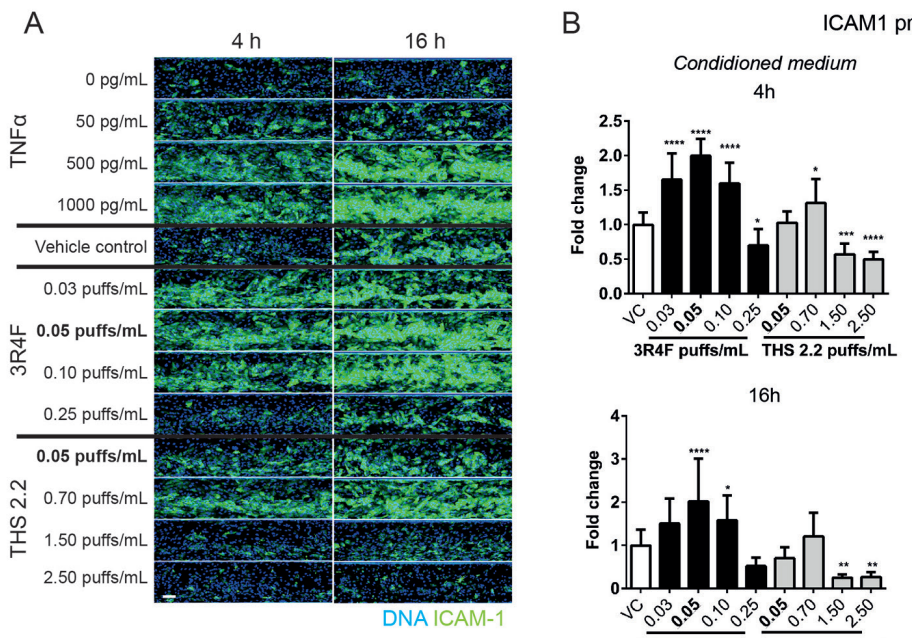

$16 \mathrm{~h}$
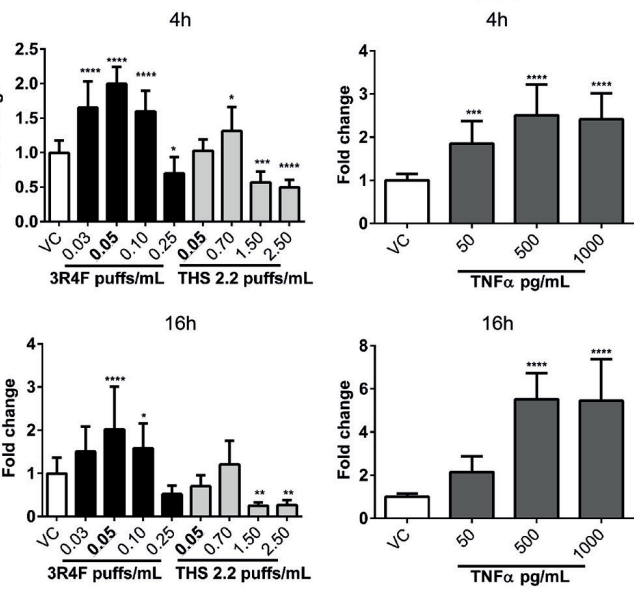

$16 \mathrm{~h}$
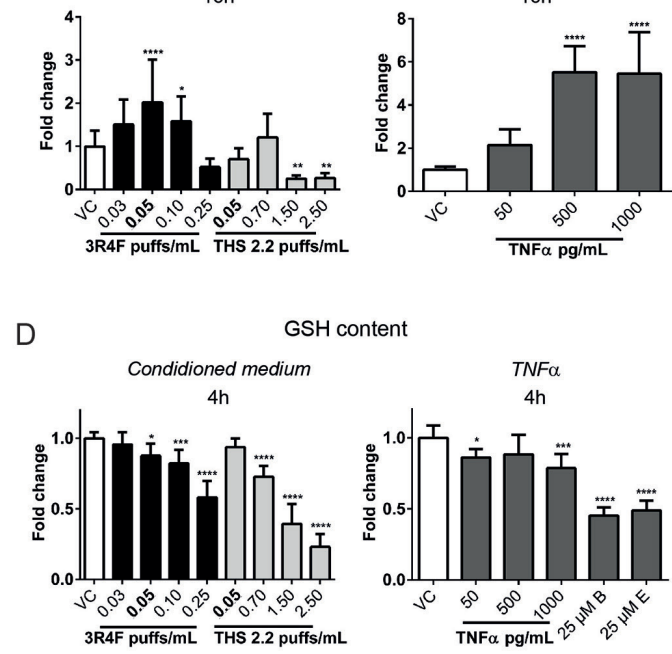

GSH content

C

$4 \mathrm{~h} \quad 16 \mathrm{~h}$

닌

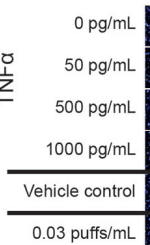

㞧 0.05 puffs $/ \mathrm{mL}$

舀

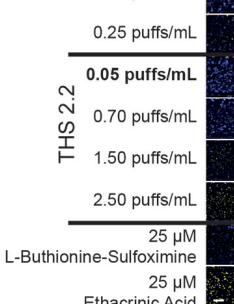

$25 \mu \mathrm{M}$

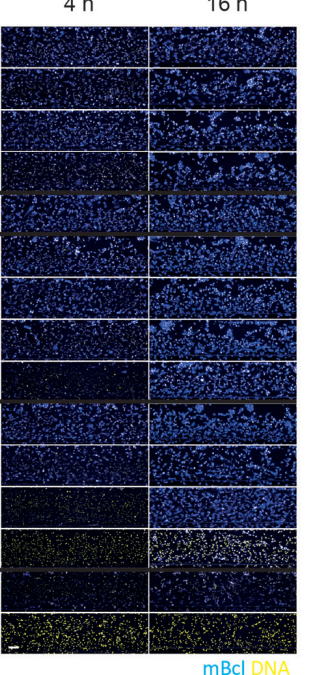

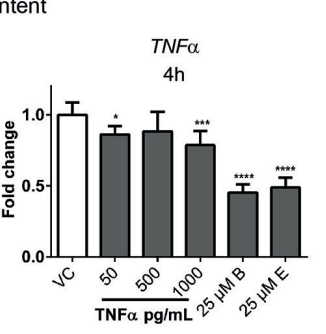
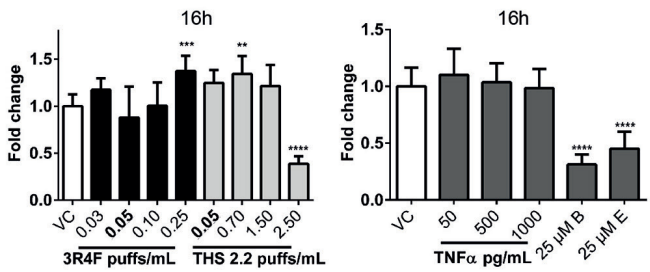

Fig. 7: 3D human microvessel-on-a-chip application for assessment of THS 2.2: quantification of key inflammatory and oxidative stress markers

HCAEC microvessels at day 4 were treated with 3R4F- or THS 2.2-conditioned media or with TNFa (50-1,000 pg/mL), L-buthioninesulfoximine $[\mathrm{B}, 25 \mu \mathrm{M}]$ and ethacrynic acid [E, $25 \mu \mathrm{M}]$ as positive controls for 4 and $16 \mathrm{~h}$. (A) Representative pictures (sum-projections) and (B) quantification barplots of ICAM1 protein in HCAEC microvessels. $N=4, n=3$. (C) Representative pictures (sum-projections) and (D) quantification barplots of GSH content in HCAEC microvessels. $\mathrm{N}=3, \mathrm{n}=3$. The results for both markers are expressed as the FC intensity (calculated per HCAEC nucleus) between treatment and its VC. ${ }^{*}, p<0.05 ;{ }^{* *}, p<0.01 ;{ }^{* \star *}, p<0.001 ;{ }^{* * \star}, p<0.0001$ (compared to VC, one-way ANOVA followed by post-hoc Dunnett's pairwise comparisons). 3R4F, reference cigarette; THS 2.2, Tobacco Heating System 2.2; ICAM1, intercellular adhesion molecule 1; GSH, glutathione

usually generated using starvation medium by reducing the percentage of FBS from $10 \%$ to $0.5 \%$. Figure $\mathrm{S}^{3}$ shows that the adaptation of HCAEC microvessels to medium with low serum content $(0.5 \%$ FBS $)$ affected HCAEC viability/proliferation and number of cells, as measured by WST- 8 enzymatic activity (panels A-D) and nuclei count (panels E-H) after 4 and $16 \mathrm{~h}$. Therefore, we decided to generate conditioned medium by maintaining the serum content at $10 \%$ FBS and to measure the levels of TNF $\alpha$ released by monocytic cells in conditioned medium as a quality check to compare with previous work done in low serum content conditions (Poussin et al., 2016, 2018).

We previously showed that $\mathrm{TNF} \alpha$, a surrogate inflammatory marker, was released by MM6 cells in the starvation medium following a 2-h treatment with 3R4F aqueous extract (Poussin 
et al., 2015). Therefore, the concentration of TNF $\alpha$ was determined in 3R4F- and THS 2.2-conditioned media containing $10 \%$ FBS (Fig. 6B). The results show a concentration-dependent increase of TNF $\alpha$ levels, reaching a maximum at $0.05 \mathrm{puffs} / \mathrm{mL}$ of $3 \mathrm{R} 4 \mathrm{~F}$ aqueous extract and then decreasing at higher concentrations $(0.1$ and $0.25 \mathrm{puffs} / \mathrm{mL})$. At the concentration of $0.05 \mathrm{puffs} /$ $\mathrm{mL}$ of $3 \mathrm{R} 4 \mathrm{~F}$ aqueous extract, at which the TNF $\alpha$ peak was measured, no significant TNF $\alpha$ release was observed for the THS 2.2 aqueous extract exposure. It was necessary to increase the concentration of THS 2.2 aqueous extract $(0.7 \mathrm{puffs} / \mathrm{mL})$ by a factor of approximately 14 to measure $\mathrm{TNF} \alpha$ levels in the conditioned medium that were similar to those obtained upon exposure to the 3R4F aqueous extract (Fig. 6B).

\subsubsection{Reduced effect of THS 2.2-conditioned medium compared with that of 3R4F-conditioned medium on MM6 cell adhesion to HCAEC microvessels} After exposure of HCAEC microvessels to 3R4F-conditioned medium for 4 and $16 \mathrm{~h}$, the adhesion of MM6 cells to the lumen of microvessels increased significantly and peaked at a concentration of $0.05 \mathrm{puffs} / \mathrm{mL}$ of $3 \mathrm{R} 4 \mathrm{~F}$ aqueous extract (Fig. $6 \mathrm{C}, \mathrm{D}$ ). At this concentration, THS 2.2-conditioned medium did not significantly promote the adhesion of MM6 cells to HCAEC microvessels (Fig. 6C, D). The concentration of THS 2.2 aqueous extract ( $0.7 \mathrm{puffs} / \mathrm{mL})$ in conditioned medium had to be enhanced by a factor of approximately 14 to yield similar adhesion (Fig. 6D). At $16 \mathrm{~h}$, no significant increase in adhesion was measured at any concentration of THS 2.2 aqueous extract in conditioned medium, and adhesion actually decreased significantly at the highest concentrations of THS 2.2 aqueous extract (1.5 and 2.5 puffs/ $\mathrm{mL}$ ) (Fig. 6D). In parallel, various concentrations of TNF $\alpha$ were included as positive controls and yielded similar results (Fig. 6C, D) to those observed (Fig. 4) in establishing the adhesion assay with HCAEC microvessels.

\subsubsection{Reduced effect of THS 2.2-conditioned medium compared with that of $3 R 4 F-c o n d i t i o n e d$ medium on ICAM 1 protein abundance and GSH content in HCAEC microvessels}

ICAM1 protein was stained and quantified in HCAEC microvessels, and patterns of ICAM1 protein abundance changes similar to those observed for MM6-HCAEC microvessel adhesion were measured after 4 or $16 \mathrm{~h}$ of exposure to conditioned medium (Fig. 7A,B). Exposure to conditioned medium did not affect the viability of the microvessel cultures, except at the highest concentration ( $2.5 \mathrm{puffs} / \mathrm{mL})$ of THS 2.2 aqueous extract (Fig. S4A ${ }^{3}$ ). The number of nuclei counted in the vessels was not affected by conditioned medium exposure (Fig. S4B ${ }^{3}$ ), indicating low toxicity of the conditioned medium at these incubation time points. In addition to soluble mediators released by MM6 cells, conditioned medium contains chemical constituents derived from cigarette smoke. We previously showed that these compounds, which remain stable in conditioned medium after a freezing/thawing cycle, can trigger oxidative stress in endothelial cells on top of an inflammatory response promoted by MM6-derived soluble mediators (Poussin et al., 2015). Therefore, we measured GSH levels to assess the magnitude of oxidative stress in HCAEC microvessels (Fig. 7C, D). We also exposed HCAEC microvessels to $25 \mu \mathrm{M}$ of L-buthionine-sulfoximine and $25 \mu \mathrm{M}$ of ethacrynic acid as positive controls. The exposure resulted in a significant reduction in GSH levels after 4 and 16 h (Fig. 7C, D). Depletion of GSH, indicating oxidative stress, was observed with increased concentrations of 3R4F or THS 2.2 aqueous extracts in conditioned medium; however, the effect on GSH levels was measured when concentrations of THS 2.2 aqueous extract in the conditioned medium were approximately 14 times higher than concentrations of 3R4F aqueous extract. After a 16-h exposure, GSH levels returned to levels similar to those of the $\mathrm{VC}$, with the exception of GSH levels in microvessels exposed to the highest concentration (2.5 puffs $/ \mathrm{mL}$ ) of THS 2.2 aqueous extract (Fig. 7C, D).

\section{Discussion}

In this work, we developed and optimized a 3D human microvessel-on-a-chip model using the OrganoPlate ${ }^{\circledR}$ platform to investigate the mechanism of leukocyte adhesion to the lumen of endothelial microvessels, a key step in the initiation of inflammation and vascular disorders, such as atherosclerosis. Moreover, we showed how this 3D endothelial vascular model can be applied to real-world applications, such as systems toxicology assessments of products in a research and development framework.

In order to establish our 3D model and grow microvessels, we used primary HCAECs, a cellular model particularly relevant for studying cardiovascular diseases. The tubular shape developed within 2 days only when endothelial cells, adherent to the ECM, were perfused, indicating that the bidirectional and oscillating flow generated by gravity-driven leveling had a mechanical effect on endothelial cells. In this context, cells intermittently undergo shear stress up to $1.6 \mathrm{dyne} / \mathrm{cm}^{2}$ when the rocker is tilted (Vormann et al., 2018). In vivo, similar-sized venules are exposed to shear stress levels between 1 and $5 \mathrm{dyne} / \mathrm{cm}^{2}$ (Cucullo et al., 2011). The endothelial barrier increased after a few hours and was formed after 2 days, remaining stable for at least 8 days. After 2 days, permeability to 4.4 and $20 \mathrm{kDa}$ dextran dropped to levels $\left(4.0 \times 10^{-5} \mathrm{~cm} / \mathrm{s}\right.$ and $3 \times 10^{-5} \mathrm{~cm} / \mathrm{s}$, respectively) comparable with those found in microvessels grown with human umbilical vein endothelial cells (van Duinen et al., 2017). HCAEC barrier formation was characterized by positive staining of the VE-cadherin and CD31 proteins, two major endothelial-junction-associated proteins that play a role in the process of blood vessel tube formation (Vestweber, 2008; Yang et al., 1999). The nature of ECM and microenvironments can influence the growth and migratory phenotypes of endothelial cells (Imanaka-Yoshida, 2016; Muncie and Weaver, 2018). Therefore, we optimized the choice of ECM and culture conditions (medium and cell density) to obtain homogeneous and reproducible HCAEC tubes devoid of cell protrusion/migration into the ECM, a characteristic of angiogenic behavior (Mongiat et al., 2016).

For functional analysis of the model, we set up an adhesion assay by flowing untreated, fluorescently labeled monocytic 
cells through the lumen of HCAEC microvessels that had been pre-stimulated with TNF $\alpha$ and quantifying the monocytic cells that remained attached to the endothelium. HCAEC microvessels responded to TNF $\alpha$ by promoting the adhesion of monocytic cells to microvessels in a concentration-dependent manner after $4 \mathrm{~h}$, with sustained effects at the highest concentrations (500 and $10,000 \mathrm{pg} / \mathrm{mL}$ ) after a 16-h treatment. In parallel, the immunohistochemical quantification of ICAM1 in HCAEC microvessels showed a correlation of the adhesion protein with monocyte-endothelium adhesion promoted by TNF $\alpha$. The kinetic profile of ICAM1 protein expression was consistent with previous findings (Granger and Senchenkova, 2010). These observations were also supported by TNF $\alpha$ concentration- and time-dependent gene expression changes, clearly showing increased transcription of inflammatory genes following the activation of the TNF $\alpha /$ nuclear factor- $\mathrm{\kappa} B$ signaling pathway. Gene sets that were significantly enriched included innate immune/inflammatory pathways such as cytokine/chemokine signaling, $\mathrm{TNF} \alpha / \mathrm{CD} 40$ signaling, and interferon $\gamma$ signaling, in addition to cell cycle processes such as DNA replication and mitosis. Interestingly, an analysis of time course protein-protein interaction networks using transcriptomics and genome-wide datasets accompanied by experimental validation has shown that $\mathrm{TNF} \alpha$ can promote $\mathrm{G} 1 / \mathrm{S}$ transition in the cell cycle in vascular endothelial cells (Chen et al., 2012). This may facilitate the cell cycle activation promoted by vascular endothelial growth factor. The comparison of the response to TNF $\alpha$ of 3D and 2D endothelial cell models showed high consistency at the levels of gene expression changes and enriched pathways/ processes. However, an accurate quantification of differences between both models would require an experiment comparing 3D and 2D cultures simultaneously, using HCAECs from one donor, which was not the case here, although our study was still robust in showing the biology perturbed by TNF $\alpha$ across independent experiments and donors. Overall, these results indicate that following TNF $\alpha$ stimulation, in vitro HCAEC microvessels can be activated and bind monocytes under flow. The pattern of adhesion correlated with the expression of adhesion molecules, similar to previous in vivo and in vitro observations in arterial and microvascular endothelial cells (Amberger et al., 1997; Jia et al., 2015; Langert et al., 2013; Mulligan et al., 1993; Zachlederova and Jarolim, 2006).

For a real-case application in systems toxicology, our HCAEC microvessel model was employed to assess the impact of aerosol from THS 2.2, a candidate MRTP, compared with the impact of $3 \mathrm{R} 4 \mathrm{~F}$ smoke in the form of conditioned medium on the process of monocytic cell adhesion to endothelial microvessels. The conditioned medium was generated by collecting the supernatant of MM6 cells exposed to aqueous extracts from 3R4F smoke or THS 2.2 aerosol for $2 \mathrm{~h}$. Conditioned medium contains a mixture of soluble mediators released by monocytic cells and chemical-derived compounds from smoke/aerosol, mimicking an in vitro milieu with inflammatory and oxidative properties, such as blood from smokers (Yanbaeva et al., 2007; Poussin et al., 2015; Zhang et al., 2002). The 4-h exposure of HCAEC microvessels to 3R4F-conditioned medium triggered an inflammatory response that promoted an increase in endothelial adhesion proteins and monocytic cell adhesion to the lumen of the HCAEC microvessels as well as endothelial oxidative stress, observed as depletion of GSH. Although the presence of serum during the exposure of HCAEC microvessels to conditioned medium may potentially reduce the effect of aqueous extracts (Grigoryan et al., 2016), these findings are similar to previous ones in HCAECs, also showing that monocyte-released soluble mediators, such as $\mathrm{TNF} \alpha$, are responsible for inducing the inflammatory response (Poussin et al., 2015; Rennert et al., 2016), while chemical compounds derived from $3 \mathrm{R} 4 \mathrm{~F}$ smoke induced oxidative stress in HCAECs (Poussin et al., 2015). At the later time point (16 h), GSH content was restored, indicating that HCAEC microvessels coped with the oxidative stress, while endothelial inflammation was still observed at some concentrations, suggesting a sustained effect of inflammatory mediators present in 3R4F-conditioned medium and/or possible autocrine feedback loops. At concentrations of 3R4F-conditioned medium at which molecular and functional effects peaked, no significant effect was observed with THS 2.2-conditioned medium. It was necessary to increase the concentration of THS 2.2 aqueous extract to generate conditioned medium by a factor of approximately 14 to record similar effects to those seen for 3R4F-conditioned medium. These results are in agreement with our previous work in a 2D HCAEC adhesion assay model (Poussin et al., 2016, 2018). Overall, this case study of employing a 3D vasculature-on-a-chip model for risk assessment in vitro shows the potential of water-soluble aerosol extract from THS 2.2 to exert a reduced impact on mechanisms that lead to the development of atherosclerosis in a pathological context, compared with extracts from cigarettes.

The microvessel model was used for acute exposure to TNF $\alpha$ or product-conditioned medium after 4 days of seeding to ensure the stability of the tube formation. Longer treatments may show different mediator and gene expression profiles more representative of the chronic conditions that lead to the development of vascular disorders. However, evaluation of endothelial microvessel barrier stability for more than 8 days will be required before conducting longer exposures.

Other microfluidic systems have been developed to study the adhesion of blood cells such as leukocytes (e.g., neutrophils), erythrocytes and platelets, and cancer cells to endothelial cells in hemodynamic flow (Conant et al., 2009; Lee et al., 2018; Thompson and Han, 2018; White et al., 2015; Lamberti et al., 2014). Various systems offer the functionality to control shear stress applied to endothelial cells, which are still, however, grown as a 2D monolayer (Conant et al., 2009; Lee et al., 2018; Thompson and Han, 2018; White et al., 2015). Interestingly, more advanced systems can be fabricated to incorporate a channel containing a stenotic structure for flow characterization (Lee et al., 2018) or to mimic an in vivo microvasculature network for studying the complete cascade of adhesion to transmigration (Lamberti et al., 2014). Nevertheless, the format of these microfluidics remains low-throughput due to the complexity of production and handling (Lee et al., 2018; Lamberti et al., 2014). A major advantage of the OrganoPlate ${ }^{\circledR}$ over other microfluidic systems is its microtiter format, which enables growing 96 independent microvessels, providing throughput capacity for multiple-condi- 
tion testing, useful for drug screening and dose-response studies. Moreover, continuous flow perfusion of media through HCAEC microvessels using gravity leveling instead of pumps facilitates handling and reduces the risk of contamination. The absence of a pump that permits the application of flow with specific shear stress magnitude and profiles (e.g., pulsatile, oscillatory) can be perceived as a limitation of the system. However, the constant evolution of the OrganoPlate ${ }^{\circledR}$ functionality and design may resolve this issue in the future. The availability of a three-lane OrganoPlate ${ }^{\circledR}$ provides significant potential to extend our HCAEC microvessel model to explore further functional aspects of vascular biology and disorders simultaneously using a single model (e.g., transmigration of monocytes). The development of co-cultures from various tissues/organs, such as smooth muscle cells, neuronal cells, or epithelial cells, paves the way to a range of potential applications. Additional experimental setups, such as concomitant exposure of leukocytes and endothelium to a treatment under flow, offer the flexibility to investigate the independent and combined contribution of both cellular types to a mechanism of interest. Moreover, perfusing human blood or serum/plasma and using endothelial cells from donors with atherosclerosis and cardiovascular disorders or donors of various ages provides an opportunity to screen a wider human population and may further contribute to the translatability of our 3D human microvessel-ona-chip model and advance its development as a precision medicine selection tool.

\section{Conclusions}

In the context of the 3 Rs principles that encourage the community to create relevant in vitro alternatives that reduce animal use, we developed and optimized a 3D vasculature-on-a-chip model using the microfluidic OrganoPlate ${ }^{\circledR}$ to investigate the process of leukocyte adhesion to the lumen of primary disease-relevant HCAEC microvessels under flow. In addition to functional readouts, we measured various molecular endpoints using high-content imaging and transcriptomics, providing mechanistic insights into the model. We demonstrated the applicability of the model for real-world research and development projects, such as systems toxicology-based risk assessment of products in vitro. The OrganoPlate ${ }^{\circledR}$ format and its design evolutions provide scalability and potential for extending the model to investigate additional mechanistic aspects of vascular diseases that would complement cardiovascular disease risk assessment and open a wide spectrum of applications in vascular biology research and beyond.

\section{References}

Ackermann, M. and Strimmer, K. (2009). A general modular framework for gene set enrichment analysis. BMC Bioinformatics 10, 47. doi:10.1186/1471-2105-10-47

Amberger, A., Maczek, C., Jurgens, G. et al. (1997). Co-expression of ICAM-1, VCAM-1, ELAM-1 and Hsp60 in human arterial and venous endothelial cells in response to cytokines and oxidized low-density lipoproteins. Cell Stress Chaperones 2, 94-
103. doi:10.1379/1466-1268(1997)002<0094:ceoive >2.3.co;2

Benjamini, Y. and Hochberg, Y. (1995). Controlling the false discovery rate: A practical and powerful approach to multiple testing. J Roy Stat Soc B 57, 289-300. doi:10.1111/j. 2517-6161.1995.tb02031.x

Bogorad, M. I., DeStefano, J., Karlsson, J. et al. (2015). Review: In vitro microvessel models. Lab Chip 15, 4242-4255. doi: $10.1039 / \mathrm{c} 51 \mathrm{c} 00832 \mathrm{~h}$

Bolstad, B. M. et al. (2005). Quality assessment of affymetrix genechip data. In R. Gentleman, V. J. Carey, W. Huber et al. (eds.), Bioinformatics and Computational Biology Solutions Using $R$ and Bioconductor. Statistics for Biology and Health. New York, NY: Springer. doi:10.1007/0-387-29362-0_3

Boue, S., Talikka, M., Westra, J. W. et al. (2015). Causal biological network database: A comprehensive platform of causal biological network models focused on the pulmonary and vascular systems. Database (Oxford) 2015, bav030. doi:10.1093/ database/bav030

Burden, N., Chapman, K., Sewell, F. et al. (2015). Pioneering better science through the 3Rs: An introduction to the national centre for the replacement, refinement, and reduction of animals in research (NC3Rs). J Am Assoc Lab Anim Sci 54, 198208.

Chatterjee, S. (2018). Endothelial mechanotransduction, redox signaling and the regulation of vascular inflammatory pathways. Front Physiol 9, 524. doi:10.3389/fphys.2018.00524

Chen, Y., Gu, J., Li, D. et al. (2012). Time-course network analysis reveals TNF-alpha can promote $\mathrm{gG} 1 / \mathrm{S}$ transition of cell cycle in vascular endothelial cells. Bioinformatics 28, 1-4. doi:10.1093/bioinformatics/btr619

Conant, C. G., Schwartz, M. A., Nevill, T. et al. (2009). Platelet adhesion and aggregation under flow using microfluidic flow cells. J Vis Exp 32, e1644. doi:10.3791/1644

Cucullo, L., Hossain, M., Puvenna, V. et al. (2011). The role of shear stress in blood-brain barrier endothelial physiology. BMC Neurosci 12, 40. doi:10.1186/1471-2202-12-40

Dai, M., Wang, P., Boyd, A. D. et al. (2005). Evolving gene/ transcript definitions significantly alter the interpretation of genechip data. Nucleic Acids Res 33, e175. doi:10.1093/nar/ gni179

Favero, G., Paganelli, C., Buffoli, B. et al. (2014). Endothelium and its alterations in cardiovascular diseases: Life style intervention. Biomed Res Int 2014, 801896. doi:10. $1155 / 2014 / 801896$

Food and Drug Administration (2012). Modified Risk Tobacco Product Applications: Draft Guidance for Industry. https:// www.fda.gov/media/83300/download

Fratta Pasini, A., Albiero, A., Stranieri, C. et al. (2012). Serum oxidative stress-induced repression of $\mathrm{Nrf} 2$ and GSH depletion: A mechanism potentially involved in endothelial dysfunction of young smokers. PLoS One 7, e30291. doi:10.1371/journal. pone. 0030291

Government of Canada (2000). Tobacco Products Information Regulations. https://www.Canada.Ca/en/health-canada/ services/health-concerns/reports-publications/tobacco/tobac co-products-information-regulations.html 
Granger, D. N. and Senchenkova, E. (2010). Inflammation and the Microcirculation. San Rafael, CA: Morgan \& Claypool Life Sciences. doi:10.4199/C00013ED1V01Y201006ISP008

Grigoryan, H., Edmands, W., Lu, S. S. et al. (2016). Adductomics pipeline for untargeted analysis of modifications to Cys34 of human serum albumin. Anal Chem 88, 10504-10512. doi:10. 1021/acs.analchem.6b02553

Haralick, R. M., Sternberg, S. R. and Zhuang, X. (1987). Image analysis using mathematical morphology. IEEE Transactions on Pattern Analysis and Machine Intelligence PAMI-9, 632550. doi:10.1109/tpami.1987.4767941

Hsieh, H. J., Liu, C. A., Huang, B. et al. (2014). Shear-induced endothelial mechanotransduction: The interplay between reactive oxygen species (ROS) and nitric oxide (NO) and the pathophysiological implications. J Biomed Sci 21, 3. doi:10.1186/ 1423-0127-21-3

Imanaka-Yoshida, K. (2016). Extracellular matrix remodeling in vascular development and disease. In T. Nakanishi, R. R. Markwald, H. S. Baldwin, B. B. Keller, D. Srivastava and H. Yamagishi (eds.), Etiology and morphogenesis of congenital heart disease: From gene function and cellular interaction to morphology. Tokyo. doi:10.1007/978-4-431-54628-3

Insull, W., Jr. (2009). The pathology of atherosclerosis: Plaque development and plaque responses to medical treatment. $\mathrm{Am} \mathrm{J}$ Med 122, S3-S14. doi:10.1016/j.amjmed.2008.10.013

Jia, Z., Nallasamy, P., Liu, D. et al. (2015). Luteolin protects against vascular inflammation in mice and TNF-alpha-induced monocyte adhesion to endothelial cells via suppressing ikappaBalpha/NF-kappaB signaling pathway. J Nutr Biochem 26, 293-302. doi:10.1016/j.jnutbio.2014.11.008

Lamberti, G., Prabhakarpandian, B., Garson, C. et al. (2014). Bioinspired microfluidic assay for in vitro modeling of leukocyte-endothelium interactions. Anal Chem 86, 8344-8351. doi: 10.1021/ac5018716

Langert, K. A., Von Zee, C. L. and Stubbs, E. B., Jr. (2013). Tumour necrosis factor alpha enhances CCL2 and ICAM-1 expression in peripheral nerve microvascular endoneurial endothelial cells. ASN Neuro 5, e00104. doi:10.1042/an20120048

Lee, J., Packard, R. R. and Hsiai, T. K. (2015). Blood flow mok dulation of vascular dynamics. Curr Opin Lipidol 26, 376-383. doi:10.1097/MOL.0000000000000218

Lee, J., Huh, H. K., Park, S. H. et al. (2018). Endothelial cell monolayer-based microfluidic systems mimicking complex in vivo microenvironments for the study of leukocyte dynamics in inflamed blood vessels. Methods Cell Biol 146, 23-42. doi:10.1016/bs.mcb.2018.05.002

Lee, S. U., Chung, S. Y. and Park, R. H. (1990). A comparative performance study of several global thresholding techniques for segmentation. Computer Vision, Graphics, and Image Processing 52, 171-190. doi:10.1016/0734-189x(90)90053-x

Ludicke, F., Picavet, P., Baker, G. et al. (2018a). Effects of switching to the menthol tobacco heating system 2.2, smoking abstinence, or continued cigarette smoking on clinically relevant risk markers: A randomized, controlled, open-label, multicenter study in sequential confinement and ambulatory settings (part 2). Nicotine Tob Res 20, 173-182. doi:10.1093/ntr/ntx028
Ludicke, F., Picavet, P., Baker, G. et al. (2018b). Effects of switching to the tobacco heating system 2.2 menthol, smoking abstinence, or continued cigarette smoking on biomarkers of exposure: A randomized, controlled, open-label, multicenter study in sequential confinement and ambulatory settings (part 1). Nicotine Tob Res 20, 161-172. doi:10.1093/ntr/ntw287

Martin, F., Sewer, A., Talikka, M. et al. (2014). Quantification of biological network perturbations for mechanistic insight and diagnostics using two-layer causal models. BMC Bioinformatics 15, 238. doi:10.1186/1471-2105-15-238

McCall, M. N., Bolstad, B. M. and Irizarry, R. A. (2010). Frozen robust multiarray analysis (fRMA). Biostatistics 11, 242-253. doi:10.1093/biostatistics/kxp059

Messner, B. and Bernhard, D. (2014). Smoking and cardiovascular disease: Mechanisms of endothelial dysfunction and early atherogenesis. Arterioscler Thromb Vasc Biol 34, 509-515. doi: 10.1161/atvbaha.113.300156

Mongiat, M.,Andreuzzi, E., Tarticchio, G. etal.(2016). Extracellular matrix, a hard player in angiogenesis. Int JMol Sci 17, doi:10. 3390/ijms17111822

Mulligan, M. S., Vaporciyan, A. A., Miyasaka, M. et al. (1993). Tumor necrosis factor alpha regulates in vivo intrapulmonary expression of ICAM-1. Am J Pathol 142, 1739-1749.

Muncie, J. M. and Weaver, V. M. (2018). The physical and biochemical properties of the extracellular matrix regulate cell fate. Curr Top Dev Biol 130, 1-37. doi:10.1016/bs.ctdb.2018. 02.002

Murphy, J., Gaca, M., Lowe, F. et al. (2017). Assessing modified risk tobacco and nicotine products: Description of the scientific framework and assessment of a closed modular electronic cigarette. Regul Toxicol Pharmacol 90, 342-357. doi:10.1016/j. yrtph.2017.09.008

Nahrendorf, M. and Swirski, F. K. (2015). Lifestyle effects on hematopoiesis and atherosclerosis. Circ Res 116, 884-894. doi:10.1161/circresaha.116.303550

Phillips, B., Veljkovic, E., Boue, S. et al. (2016). An 8-month systems toxicology inhalation/cessation study in apoe-/- mice to investigate cardiovascular and respiratory exposure effects of a candidate modified risk tobacco product, ths 2.2, compared with conventional cigarettes. Toxicol Sci 149, 411-432. doi:10.1093/toxsci/kfv243

Poussin, C., Laurent, A., Peitsch, M. C. et al. (2015). Systems biology reveals cigarette smoke-induced concentration-dependent direct and indirect mechanisms that promote monocyte-endothelial cell adhesion. Toxicol Sci 147, 370-385. doi:10. 1093/toxsci/kfv137

Poussin, C., Laurent, A., Peitsch, M. C. et al. (2016). Systems toxicology-based assessment of the candidate modified risk tobacco product THS2.2 for the adhesion of monocytic cells to human coronary arterial endothelial cells. Toxicology 339, 73 86. doi:10.1016/j.tox.2015.11.007

Poussin, C., Laurent, A., Kondylis, A. et al. (2018). In vitro systems toxicology-based assessment of the potential modified risk tobacco product CHTP 1.2 for vascular inflammation- and cytotoxicity-associated mechanisms promoting adhesion of monocytic cells to human coronary arterial endothelial cells. 
Food Chem Toxicol 120, 390-406. doi:10.1016/j.fct.2018. 07.025

Radeva, M. Y. and Waschke, J. (2018). Mind the gap: Mechanisms regulating the endothelial barrier. Acta Physiol (Oxf) 222, doi:10.1111/apha. 12860

Rennert, K., Heisig, K., Groeger, M. et al. (2016). Recruitment of $\mathrm{CD} 16(+)$ monocytes to endothelial cells in response to LPS-treatment and concomitant TNF release is regulated by CX3CR1 and interfered by soluble fractalkine. Cytokine 83, 41-52. doi:10.1016/j.cyto.2016.03.017

sbv IMPROVER project team, Ansari, S., Binder, J. et al. (2013). On crowd-verification of biological networks. Bioinform Biol Insights 7, 307-325. doi:10.4137/BBI.S12932

Schaller, J. P., Keller, D., Poget, L. et al. (2016). Evaluation of the tobacco heating system 2.2. Part 2: Chemical composition, genotoxicity, cytotoxicity, and physical properties of the aerosol. Regul Toxicol Pharmacol 81 Suppl 2, S27-S47. doi:10.1016/j. yrtph.2016.10.001

Smyth, G. K. (2004). Linear models and empirical bayes methods for assessing differential expression in microarray experiments. Stat Appl Genet Mol Biol 3, Article3. doi:10.2202/15446115.1027

Sternberg (1983). Biomedical image processing. Computer 16, 22-34. doi:10.1109/MC.1983.1654163

Subramanian, A., Tamayo, P., Mootha, V. K. et al. (2005). Gene set enrichment analysis: A knowledge-based approach for interpreting genome-wide expression profiles. Proc Natl Acad Sci U S A 102, 15545-15550. doi:10.1073/pnas.0506580102

Surgeon General (2010). How tobacco smoke causes disease: The biology and behavioral basis for smoking-attributable disease: A report of the surgeon general. Atlanta (GA). doi:10.1037/e590462011-001

Thompson, T. J. and Han, B. (2018). Analysis of adhesion kinetics of cancer cells on inflamed endothelium using a microfluidic platform. Biomicrofluidics 12, 042215. doi:10. 1063/1.5025891

van der Toorn, M., Frentzel, S., De Leon, H. et al. (2015). Aerosol from a candidate modified risk tobacco product has reduced effects on chemotaxis and transendothelial migration compared to combustion of conventional cigarettes. Food Chem Toxicol 86, 81-87. doi:10.1016/j.fct.2015.09.016

van Duinen, V., van den Heuvel, A., Trietsch, S. J. et al. (2017). 96 perfusable blood vessels to study vascular permeability in vitro. Sci Rep 7, 18071. doi:10.1038/s41598-017-14716-y

Vestweber, D. (2008). Ve-cadherin: The major endothelial adhesion molecule controlling cellular junctions and blood vessel formation. Arterioscler Thromb Vasc Biol 28, 223-232. doi:10.1161/atvbaha.107.158014
Vormann, M. K., Gijzen, L., Hutter, S. et al. (2018). Nephrotoxicity and kidney transport assessment on 3D perfused proximal tubules. AAPS J 20, 90. doi:10.1208/s12248-018-0248-z

White, J., Lancelot, M., Sarnaik, S. et al. (2015). Increased erythrocyte adhesion to VCAM-1 during pulsatile flow: Application of a microfluidic flow adhesion bioassay. Clin Hemorheol Microcirc 60, 201-213. doi:10.3233/ch-141847

Yanbaeva, D. G., Dentener, M. A., Creutzberg, E. C. et al. (2007). Systemic effects of smoking. Chest 131, 1557-1566. doi:10.1378/chest.06-2179

Yang, S., Graham, J., Kahn, J. W. et al. (1999). Functional roles for PECAM-1 (CD31) and ve-cadherin (CD144) in tube assembly and lumen formation in three-dimensional collagen gels. Am J Pathol 155, 887-895. doi:10.1016/s0002-9440 (10)65188-7

Zachlederova, M. and Jarolim, P. (2006). The dynamics of gene expression in human lung microvascular endothelial cells after stimulation with inflammatory cytokines. Physiol Res 55, $39-47$.

Zhang, X., Wang, L., Zhang, H. et al. (2002). The effects of cigarette smoke extract on the endothelial production of soluble intercellular adhesion molecule-1 are mediated through macrophages, possibly by inducing TNF-alpha release. Methods Find Exp Clin Pharmacol 24, 261-265. doi:10.1358/mf.2002.24.5.802302

\section{Conflict of interest}

This publication contains original work. The authors CP, AL, DP, KB, RD, EG, NVI, MCP and JH are employees of Philip Morris International. The authors BK, HL, TO, MV, AvdH and JJ are or were employees of MIMETAS BV. These affiliations are declared. The OrganoPlate ${ }^{\circledR}$ is a registered trademark of MIMETAS BV.

\section{Acknowledgements}

Philip Morris International is the sole source of funding and sponsor of this research. We thank Pauline Betsch and Dr Remko van Vught for project management support, Didier Goedertier and Claudius Pak for generating the aqueous smoke/aerosol extracts, Dr Alain Sewer for preparing and submitting transcriptomics data to the Array Express repository database, Dr Stéphanie Boué for preparing and publishing the study data in INTERVALS (https:// www.intervals.science/), and Frederik Schavemaker for support with schematic illustrations. We also thank Dr Dean Meyer from Edanz Medical Writing (https://www.edanzediting.com/ac) for editing a draft of this manuscript. 